\title{
7. GEOCHEMISTRY OF THE LOWER SHEETED DIKE COMPLEX, HOLE 504B, LEG 140 ${ }^{1}$
}

\author{
Joel W. Sparks ${ }^{2}$
}

\begin{abstract}
Sixty-three samples representing $379 \mathrm{~m}$ of sheeted dikes from Deep Sea Drilling Project/Ocean Drilling Program Site 504B have been analyzed for major and selected trace elements by X-ray fluorescence. The samples range from microcrystalline aphyric basalts to moderately phyric ( $2 \%-10 \%$ phenocrysts) diabase that are typically multiply saturated with plagioclase, olivine, and clinopyroxene, in order of relative abundance. All analyzed samples are classified as Group D compositions with moderate to slightly elevated compatible elements $\left(\mathrm{Mg}^{\prime}\right.$-value $=0.65 \% \pm 0.03 \% ; \mathrm{Al}_{2} \mathrm{O}_{3}=15.5 \% \pm 0.8 \% ; \mathrm{CaO}=13.0 \% \pm 0.3 \% ; \mathrm{Ni}=114$ $\pm 29 \mathrm{ppm}$ ), and unusually depleted levels of moderate to highly incompatible elements ( $\mathrm{Nb} \leq 1 \mathrm{ppm} ; \mathrm{Zr}=44 \pm 7 \mathrm{ppm} ; \mathrm{Rb}<0.5$ $\mathrm{ppm} ; \mathrm{Ba} \approx 1 \mathrm{ppm} ; \mathrm{P}_{2} \mathrm{O}_{5}=0.07 \% \pm 0.02 \%$ ). These compositions are consistent with a multistage melting of a normal ocean ridge basaltic mantle source followed by extensive fractionation of olivine, plagioclase, and clinopyroxene.

Leg 140 aphyric to sparsely phyric (0\%-2\% phenocrysts) basalts and diabases are compositionally indistinguishable from similarly phyric samples at higher levels in the hole. An examination of the entire crustal section, from the overlying volcanics through the sheeted dikes observed in Leg 140, reveals no significant trends indicating the enrichment or depletion of Costa Rica Rift Zone source magmas over time. Similarly, significant trends toward increased or decreased differentiation cannot be identified, although compositional patterns reflecting variable amounts of phenocryst addition are apparent at various depths. Below $\approx 1700 \mathrm{mbsf}$ to the bottom of the Leg 140 section, there is a broadly systematic pattern of $\mathrm{Zn}$ depletion with depth, the result of high-temperature hydrothermal leaching. This zone of depletion is thought to be a significant source of $\mathrm{Zn}$ for the hydrothermal fluids depositing metal sulfides at ridge-crest hydrothermal vents and the sulfide-mineralization zone, located in the transition between pillow lavas and sheeted dikes.

Localized zones of intense alteration ( $60 \%-95 \%$ recrystallization) are present on a centimeter to meter scale in many lithologic units. Within these zones, normally immobile elements $\mathrm{Ti}, \mathrm{Zr}$, Y, and rare-earth elements are strongly depleted compared with "fresher" samples centimeters away. The extent of compositional variability of these elements tends to obscure primary igneous trends if the highly altered samples are not identified or removed. At levels up to $40 \%$ (or possibly $60 \%$ ) recrystallization, $\mathrm{Ti}, \mathrm{Zr}$, and $\mathrm{Y}$ retain their primary signatures. Although the mechanisms are unclear, it is possible that these intense alteration zones are a source of $\mathrm{Y}$ and rare-earth elements for the typically rare-earth-element-enriched hydrothermal vent fluids of mid-ocean ridges.
\end{abstract}

\section{INTRODUCTION AND GEOLOGIC SETTING}

Leg 140 marked the sixth excursion of the Deep Sea Drilling Project/Ocean Drilling Program (DSDP/ODP) to core Hole 504B, located approximately $200 \mathrm{~km}$ south of the Costa Rica Rift Zone $\left(1^{\circ} 13.611^{\prime} \mathrm{N}, 83^{\circ} 43.818^{\prime} \mathrm{W}\right)$. The $379 \mathrm{~m}$ drilled during this leg deepened the hole to a total depth of 2000.4 meters below seafloor (mbsf), making it the deepest penetration into oceanic crust (1725.9 m). Hole 504B has already provided an unprecedented view of the oceanic crust as it has penetrated $572 \mathrm{~m}$ of extrusive volcanics (Layer $2 \mathrm{~A}$ ), $209 \mathrm{~m}$ of a mixed extrusive/sheeted dike transition zone (Layer 2B), and $945 \mathrm{~m}$ of sheeted dikes (Layer 2C). The main intent of Leg 140 was to drill through and sample the Layer $2 \mathrm{C}-3$ transition, from sheeted dikes to layered gabbros, the existence of which is inferred from analogies to ophiolite complexes (e.g., Coleman, 1977) and to plutonic rock sequences sampled in submarine fracture zones (e.g., Von Herzen, Robinson, et al., 1991).

The Costa Rica Rift Zone (CRRZ) is an intermediate-rate spreading ridge on the easternmost extremity of the Cocos-Nazca Spreading Center, bounded by the Ecuador Fracture Zone to the west, and the Panama Fracture Zone to the east (Hey et al., 1977; Lonsdale and Klitgord, 1978). The asymmetric spreading rates of $3.6 \mathrm{~cm} / \mathrm{yr}$ to the

'Erzinger, J., Becker, K., Dick, H.J.B., and Stokking, L.B. (Eds.), 1995, Proc, ODP, Sci. Results, 137/140: College Station, TX (Ocean Drilling Program).

2Department of Geology, Stanford University, Stanford, CA 94305, U.S.A. south and $3.0 \mathrm{~cm} / \mathrm{yr}$ to the north (Hey et al., 1977) are consistent with the characteristically uniform seafloor topography of an intermediate-rate ridge. Hole 504B is situated between magnetic Anomalies $3 \mathrm{~A}$ and 4 , in 5.9-m.y.-old oceanic crust.

The basalts and diabases from Hole 504B are typically multiply saturated with respect to olivine, plagioclase, and clinopyroxene, and range from $0 \%$ to $10 \%$ phenocrysts, of which plagioclase is the most common. Considerable work from previous legs has focused on characterizing Hole 504B igneous compositions based on bulk-rock major and trace element analyses (Autio and Rhodes, 1983; Etoubleau et al., 1983; Hubberten et al., 1983; Marsh et al., 1983; Sharaskin et al., 1983; Emmermann, 1985; Kempton et al., 1985; Tual et al., 1985; Autio et al., 1989), isotopic determinations (Barrett and Friedrichsen, 1982; Barrett, 1983; Friedrichsen, 1985; Kusakabe et al., 1989; Shimizu et al., 1989), and electron microprobe analyses of fresh glasses and selected mineral phases (Natland, et al., 1983; Kempton, 1985; Kempton et al., 1985). Using the classification scheme of Autio and Rhodes (1983) and Kempton et al. (1985), over 98\% of the samples analyzed from this location are Group D (depleted) compositions. The remaining $2 \%$ belong to either Groups $\mathrm{M}$ (moderately evolved), E (enriched), or T (transitional). Groups $\mathrm{M}$ and $\mathrm{E}$ correspond to normal Type I and Type II mid-ocean-ridge basalt (MORB) compositions (e.g., Bryan et al., 1976), whereas Group T is transitional between them. Group D bulk-rock and glass compositions vary from slightly olivine- to quartz-normative tholeiites, and are similar in many respects (compatible major- and trace-element abundances; $\mathrm{Sr}$ and $\mathrm{Nd}$ isotopes) to moderately evolved, normal Type I MORB. 
Despite these similarities, Group D compositions exhibit marked levels of depletion in moderate to highly incompatible element abundances relative to Type I MORB. These characteristics have been modeled as either unusual primitive melt compositions (Natland et al., 1983), or the result of multistage melting of a normal MORB mantle source, followed by extensive crystal fractionation (Autio and Rhodes, 1983; Autio, 1984; Kempton et al., 1985; Autio et al., 1989; Kusakabe et al., 1989; Shimizu et al., 1989).

Nearly all samples recovered from the sheeted dike section show mineralogical and compositional signs of subsolidus alteration ranging from slight $(<10 \%)$ to extensive $(95 \%)$ recrystallization. These changes result from a complex interplay between alteration under greenschist facies conditions $\left(250^{\circ}-380^{\circ} \mathrm{C}\right)$ near the ridge axis, precipitation of anhydrite from deep penetrating seawater as the crust moved off-axis into recharge zones, and low-temperature formation of zeolites $\left(\leq 150^{\circ} \mathrm{C}\right)$ as the section moved further off-axis (Alt, 1984; Alt et al., 1985, 1986a, 1989b; Shipboard Scientific Party, 1992). Bulk-rock compositional changes that take place during alteration have been documented at Hole 504B for major elements (Honnorez et al., 1983; Hubberten et al., 1983; Noack et al., 1983; Alt and Emmermann, 1985; Emmermann, 1985; Kawahata and Furuta, 1985; Alt et al., 1989b), selected trace elements (Belyi et al., 1983; Hubberten, 1983; Hubberten et al., 1983; Alt and Emmermann, 1985; Kawahata and Furuta, 1985; Alt et al., 1989a, 1989b), as well as stable and radiogenic isotopes (Barrett, 1983; Barrett and Friedrichsen, 1982, 1983; Belyi et al., 1983; Honnorez et al., 1983; Friedrichsen, 1985; Mitchell and Terrell, 1985; Alt et al., 1986a, 1986b, 1989a, 1989b; Alt and Chaussidon. 1989; Kusakabe et al., 1989; Shimizu et al., 1989; Ishikawa and Nakamura, 1992). These compositional variations are controlled by the style of alteration, which in turn, is dependent upon the given section of oceanic crust that is of interest. Lowtemperature $\left(0^{\circ}-100^{\circ} \mathrm{C}\right)$, high water/rock ratio $(10-100)$ seawater alteration dominates the highly porous pillow lava section down to the beginning of the transition into the sheeted dikes. Below the transition, higher temperatures $\left(200^{\circ}-380^{\circ} \mathrm{C}\right.$; greenschist facies mineralogy) and significantly lower water/rock ratios (1-5), characterize the hydrothermal alteration in the sheeted dikes. Basalts in this section are heterogeneously altered (on a scale of centimeters to tens or hundreds of meters), exhibiting trends toward losses of $\mathrm{SiO}_{2}, \mathrm{CaO}, \mathrm{TiO}_{2}$; variable changes in $\mathrm{Al}_{2} \mathrm{O}_{3}$; gains in $\mathrm{MnO}, \mathrm{Na}_{2} \mathrm{O}, \mathrm{CO}_{2}, \mathrm{H}_{2} \mathrm{O}^{+}, \mathrm{S}, \mathrm{MgO}$ (slight), and oxidation of $\mathrm{Fe}$; and some mobility of light rare-earth elements (Alt and Emmermann, 1985).

$\mathrm{Ti}, \mathrm{Zr}$, and $\mathrm{Y}$ are generally considered to be relatively immobile elements and extremely useful for evaluating igneous processes in basalts that have been exposed to a range of subsolidus alteration processes since initial emplacement (e.g., Cann, 1970; Winchester and Floyd, 1976; Humphris and Thompson, 1978; Pearce and Norry, 1979). However, recent work on the secondary alteration of basalts (e.g., Bienvenu et al., 1990; Price et al., 1991) has shown that these elements are not always immobile, and the preliminary findings of $\mathrm{Ti}, \mathrm{Zr}$, and $\mathrm{Y}$ depletion in localized areas of intense alteration (Shipboard Scientific Party, 1992), appear to support this notion.

The main goals of this study are to document and discuss the variations in primary igneous chemistry of the sheeted dike complex, as the hole approaches the Layer 3 gabbros, and to document the variations of $\mathrm{Ti}, \mathrm{Zr}, \mathrm{Y}$, and selected rare-earth elements (REE) due to hydrothermal alteration. Particular emphasis is placed on how these variations affect normal primary igneous trends. Finally, a discussion is presented concerning the presence and implications of a $\mathrm{Zn}$ depletion zone near the base of the Leg 140 section.

\section{SAMPLING AND ANALYTICAL METHODS}

Most samples used in this study were chosen as being representative of the lithologic units described by the Shipboard Scientific Party
(1992), including three samples from the Leg 137 core $(8.8 \mathrm{~m}$ total recovery). In addition, several lithologic units displaying a wide range of hydrothermal alteration were sampled in detail to document the compositional variability of $\mathrm{Ti}, \mathrm{Zr}, \mathrm{Y}$, and REE resulting from subsolidus alteration.

Prior to analysis, samples were trimmed and ground to remove any large ( $>0.5 \mathrm{~mm}$ wide) hydrothermal veins and outside surfaces that may have been contaminated by exposure to the coring bit. Saw marks were removed on a silicon carbide grinding wheel, and samples were cleaned with distilled water in an ultrasonic bath. Cleaned rock chips were reduced in a tungsten carbide (WC) jaw crusher, and ground to a powder ( $\leq 200$ mesh) in a $20 \mathrm{~mL}$ WC-shatterbox (graciously lent to the author by J.M. Rhodes, University of Massachusetts). The amount of powder produced for each sample ranged from 8 to $43 \mathrm{~g}$, with most samples over $15 \mathrm{~g}$. Powders to be used for major element analysis were ignited for 4 to $6 \mathrm{hr}$ at $1000^{\circ} \mathrm{C}$ to determine loss on ignition and to oxidize iron to the trivalent state. Duplicate glass discs for each sample were prepared for X-ray fluorescence (XRF) major element analysis by fusing ignited sample powder with a lanthanum-bearing lithium borate flux (Norrish and Hutton, 1969). Samples for XRF trace analysis were prepared with approximately 5 $\mathrm{g}$ of unfired sample powder pressed into a boric acid backing using a hydraulic press (Norrish and Chappell, 1977). Major and trace element ( $\mathrm{Nb}, \mathrm{Zr}, \mathrm{Y}, \mathrm{Sr}, \mathrm{Rb}, \mathrm{Ga}, \mathrm{Zn}, \mathrm{Cu}, \mathrm{Ni}, \mathrm{Cr}, \mathrm{V}, \mathrm{Cl}$, and $\mathrm{S}$ ) abundances were analyzed at Stanford University using an automated Rigaku SMAX wavelength-dispersive spectrograph, equipped with an endwindow, Rh-target X-ray tube. Major element matrix corrections were made following the method of Norrish and Hutton (1969). Because ignited powders were used, all major element analyses are reported on an anhydrous basis, with total iron as $\mathrm{Fe}_{2} \mathrm{O}_{3}\left(\mathrm{Fe}_{2} \mathrm{O}_{3}{ }^{\circ}\right)$. Loss on ignition $\left(\mathrm{LOI}^{\circ}\right)$ is corrected for the oxidation of $\mathrm{FeO}$ to $\mathrm{Fe}_{2} \mathrm{O}_{3}$ (assuming the average $\mathrm{FeO}$ content of a typically altered sample is $85 \%$ of the total iron content). Trace element analyses were corrected for nonlinear backgrounds and spectral interferences after Norrish and Chappell (1977). Matrix absorption effects for all XRF trace elements except $\mathrm{Cl}$ and $\mathrm{S}$ were measured using a modified Compton scattering method (Reynolds, 1963; 1967; Walker, 1973; Willis, 1989). Mass absorption coefficients for $\mathrm{S}$ and $\mathrm{Cl}$ were calculated from major element analyses using mass absorption data tables (e.g., Norrish and Chappell, 1977). All XRF analyses are presented in Table 1. Estimates of accuracy and precision (Table 2) were determined by analyzing well-characterized basalt and diabase standards (USGS standards BIR-1 and DNC-1, and ODP Leg 111 inter-laboratory standard BAS-111) together with the unknowns. These standards were chosen for their broad compositional similarities to the highly depleted Leg 140 diabase to provide a realistic assessment of analytical precision.

Using compositions determined by XRF as the basis for selection, rare-earth elements ( $\mathrm{La}, \mathrm{Ce}, \mathrm{Pr}, \mathrm{Nd}, \mathrm{Sm}, \mathrm{Eu}, \mathrm{Gd}, \mathrm{Tb}, \mathrm{Dy}, \mathrm{Ho}, \mathrm{Er}, \mathrm{Tm}$, $\mathrm{Yb}$, and $\mathrm{Lu}$ ) and $\mathrm{Ba}$ were determined on a subset of samples by inductively coupled plasma mass spectrometry (ICPMS) at Union College, Schenectady, New York, by J. Tepper. Unfired sample powders were digested using an $\mathrm{HNO}_{3}-\mathrm{HF}$ digestion technique, followed by dilution with $0.1 \mathrm{~N} \mathrm{HNO}_{3}$. Internal standards (Cs and Ta) were added to the solutions for monitoring instrumental drift. All samples were analyzed in duplicate, and the resulting concentrations were calibrated against basalt standard NBS-688. Estimates of precision were made using the formula

$$
s=\sqrt{\Sigma(x-\bar{x})^{2} /(n-1)}
$$

where $x$ and $\bar{x}$ are the observation and mean for replicate analyses of the same element in one sample, and $n$ is the number of samples analyzed (e.g., Autio and Rhodes, 1983). This method produces error 
estimates that reflect the true errors associated with the concentration levels observed in the Leg 140 diabases. Basalt and diabase standards BIR-I and DNC-I were analyzed together with the unknowns as a measure of accuracy. The ICPMS analyses and analytical statistics are presented in Table 3.

Various authors have reported significant sample contamination for $\mathrm{W}, \mathrm{Co}, \mathrm{Ta}$, and to a lesser extent, $\mathrm{Nb}$ in samples that are ground in a WC shatterbox (Thompson and Bankston, 1970; Joron et al., 1980; Hickson and Juras, 1986). Joron et al. (1980) observed an average $2 \mathrm{ppm}$ increase in $\mathrm{Nb}$ content of basalts ground in WC vs. those ground in agate, whereas Hickson and Juras (1986) suggest a smaller increase $(\approx 0.5 \mathrm{ppm})$ under similar conditions. In the present study, $\mathrm{Nb}$ contamination does not appear to be significant $(<<1 \mathrm{ppm})$, because the maximum measured concentration is only $1.0 \mathrm{ppm}$, and many of the analyzed samples are close to the lower limit of detection $(0.3 \mathrm{ppm} ; 2 \sigma$ counting error). This reduced level of contamination may be due, in part, to decreased sample hardness resulting from subsolidus alteration, relatively short grinding times $(\approx 90 \mathrm{~s})$, or the use of a "cleaner" tungsten carbide in the fabrication of the shatterbox used in this study (Spex Industries, Inc., pers. comm., 1992).

Two terms referred to throughout this study are the "degree of alteration" and $\mathrm{Mg}^{\prime}$-value. The degree of alteration is the total percentage of secondary mineralization as determined by either thin-section point counts or visual inspection of sliced core samples (Shipboard Scientific Party, 1992). A rough estimate of accuracy and precision is approximately $\pm 10 \%$ (absolute). $\mathrm{Mg}^{\prime}$-value is the molar ratio $\mathrm{MgO}$ / $\left(\mathrm{MgO}+\mathrm{FeO}\right.$ ), where $\mathrm{Fe}^{2+} /\left(\mathrm{Fe}^{2+}+\mathrm{Fe}^{3+}\right)$ is equal to 0.90 (Basaltic Volcanism Study Project, 1981).

\section{IGNEOUS CHEMISTRY}

The mineralogy, water content, and the abundance of normally mobile elements ( $\mathrm{Na}, \mathrm{K}, \mathrm{Rb}, \mathrm{Sr}, \mathrm{Cu}, \mathrm{S}$, and $\mathrm{Cl}$ ) indicate that all samples selected for this study have undergone varying degrees of hydrothermal alteration. The effects of this alteration must be quantified to assess primary igneous trends, many of which could be partially or fully obscured by compositional variations. To this end, samples that have undergone more than $60 \%$ recrystallization of their groundmass phases are arbitrarily classified as "highly altered," and will not be used in discussions of igneous compositions unless specifically mentioned. The selection of this alteration indicator is discussed later in this study.

The following descriptions and discussion of igneous compositions include 74 samples analyzed by the Shipboard Scientific Party (1992), using analytical methods and calibration standards similar to this study. For comparison, 10 of the analyzed shipboard samples were measured at Stanford, and the mean differences between the two data sets are listed in Table 2. Only $\mathrm{Zn}$ and $\mathrm{Cr}$ exhibit significant deviations ( 11 and $12 \mathrm{ppm}$ lower than this study, respectively), and each shipboard analysis has been adjusted accordingly for more precise comparisons. Data selected from previous legs to Hole 504B were produced at the University of Massachusetts (Autio and Rhodes 1983; Kempton, et al., 1985; Autio et al., 1989). The Leg 111 interlaboratory standard, analyzed at the University of Massachusetts (Autio et al., 1989) and in this study, is listed in Table 2 for comparison.

All Leg 137/140 dike samples can be classified as Group D compositions (Autio and Rhodes, 1983; Kempton et al., 1985), with moderate to slightly elevated levels of compatible elements $(\mathrm{MgO}=$ $7.7 \%-10.0 \% ; \mathrm{Fe}_{2} \mathrm{O}_{3}{ }^{*}=8.1 \%-11.7 \% ; \mathrm{CaO}=11.7 \%-13.8 \% ; \mathrm{Al}_{2} \mathrm{O}_{3}=$ $14.2 \%-17.9 \% ; \mathrm{Ni}=74-189 \mathrm{ppm} ; \mathrm{Cr}=127-456 \mathrm{ppm} ; \mathrm{V}=174-374$ ppm), unusually high $\mathrm{CaO} / \mathrm{Na}_{2} \mathrm{O}$ ratios (up to 9.6), and highly depleted concentrations of moderately to highly incompatible elements $\left(\mathrm{TiO}_{2}=0.67 \%-1.14 \% ; \mathrm{K}_{2} \mathrm{O} \approx 0.01 \% ; \mathrm{P}_{2} \mathrm{O}_{5}=0.04 \%-0.13 \% ; \mathrm{Nb} \leq 1\right.$ $\mathrm{ppm} ; \mathrm{Zr}=31-62 \mathrm{ppm} ; \mathrm{Sr}=43-77 \mathrm{ppm} ; \mathrm{Rb}<0.5 \mathrm{ppm}$ ).
Analyzed samples typically fall into three petrographic classes: microcrystalline to fine-grained aphyric basalt ( $\leq 1 \%$ phenocrysts), fine- to medium-grained sparsely phyric diabase $(1 \%-2 \%$ phenocrysts), and fine- to medium-grained, moderately phyric diabase ( $2 \%-$ $10 \%$ phenocrysts). Although there is significant compositional overlap, from aphyric to porphyritic samples (Table 4), small increases in $\mathrm{Al}_{2} \mathrm{O}_{3}(15.0 \%-15.9 \%)$, Ni (98-119 ppm), $\mathrm{Cr}(271-337 \mathrm{ppm})$, and decreases in $\mathrm{TiO}_{2}(0.99 \%-0.86 \%), \mathrm{Fe}_{2} \mathrm{O}_{3}{ }^{*}(10.5 \%-9.7 \%), \mathrm{Zr}(51-42$ $\mathrm{ppm}), \mathrm{Y}(26-22 \mathrm{ppm})$, and $\mathrm{V}(314-274 \mathrm{ppm})$ reflect the addition of olivine, plagioclase, $(\mathrm{Cr}$-rich) clinopyroxene, and $\mathrm{Cr}$-spinel components.

The average composition of aphyric to sparsely phyric samples from Leg $137 / 140$ is nearly identical to that of the Group D basalts from Layer 2B-2C transition zone-sheeted dikes (Legs 83 and 111), and the Layer 2A volcanic section of Legs 69 and 70, provided normally mobile elements, $\mathrm{K}$ and $\mathrm{Rb}$, are discounted (Table 5). The aphyric samples are olivine-normative tholeiites with $\mathrm{Mg}^{\prime}$-values $(0.60$ 0.70 ) and $\mathrm{Ni}$ abundances (74-143 ppm) similar to most moderately evolved mid-ocean-ridge basalts (Figs. 1 and 2 ). In contrast to most MORB, however, these compositions are markedly lower in $\mathrm{TiO}_{2}$ and $\mathrm{Zr}$ at a given level of evolution such as $\mathrm{Mg}^{\prime}$-value or $\mathrm{Ni}$ abundance. Included in Figures 1 and 2 is Sample 111-504B-159R-1, 52$56 \mathrm{~cm}$ (Piece 9), the most evolved Group D composition ( $\mathrm{Mg}^{\prime}$-value $=0.56 ; \mathrm{Ni}=55 \mathrm{ppm}$ ) observed at Hole 504B (Autio et al., 1989). A 1-atm fractionation path calculated using the computer program CHAOS (e.g., Nielsen, 1985, 1990) is shown for comparison. The starting composition was primitive aphyric Sample 69-504B-35R-1, $100 \mathrm{~cm}\left(\mathrm{Mg}^{\prime}\right.$-value $=0.70$; Autio and Rhodes, 1983), and fractionation increments of $2 \%$ were used (see also, Naslund et al., this volume). Based on this path, the range of observed compositions represents roughly $35 \%$ fractionation of olivine + plagioclase, followed by olivine + plagioclase + clinopyroxene. These calculations agree closely with fractionation paths based on experimental (1 atm) melts of Group D Costa Rica Rift Zone compositions of Autio (1984).

Group $\mathrm{D}$ compositions, characterized by $\mathrm{Zr}$ and $\mathrm{Nb}$ concentrations that are extremely depleted relative to Type I MORB (i.e., Group M) at similar stages of differentiation, have $\mathrm{Zr} / \mathrm{Nb}$ ratios that are similar to Type I basalts (Autio and Rhodes, 1983; Kempton et al., 1985; Autio et al., 1989). Figure 3 shows that all Leg 140/137 dike samples fall into the same category - depleted incompatible element signature and $\mathrm{Zr} / \mathrm{Nb}$ ratios greater than 30 . Figure 4 depicts variations in $\mathrm{Zr}$ and $\mathrm{Zr} / \mathrm{Y}$ for the basalts and diabases of Hole $504 \mathrm{~B}$ compared with other mid-ocean-ridge basalts. Most Hole 504B samples are highly depleted in $\mathrm{Zr}$ and $\mathrm{Zr} / \mathrm{Y}$ with respect to typical midocean-ridge basalts, and this level of depletion is not significantly affected by simple multiphase fractionation paths of Autio et al. (1989).

\section{COMPOSITIONAL VARIATIONS WITH DEPTH}

The incompatible element signatures of the Leg 137/140 dike samples are relatively uniform between 1620 mbsf and $2000 \mathrm{mbsf}$ (Fig. 5). Levels of $\mathrm{Nb}(\leq 1 \mathrm{ppm}), \mathrm{Zr}(44 \pm 7 \mathrm{ppm})$, and $\mathrm{Zr} / \mathrm{Y}(1.9 \pm 0.2)$ are well within the typical ranges defined by previous legs to this site. Moreover, when samples from the entire hole are considered, there are no significant systematic trends toward enrichment or depletion of incompatible elements with depth. Similarly, there are no systematic differentiation trends with depth, as indicated by compatible elements (Figs. 6 and 7). Instead, there are regions of increased compositional variability (at approximately 1300 and $1700 \mathrm{mbsf}$ ) separated by a section of minimal variation, near $1550 \mathrm{mbsf}$. This pattern is consistent with variable addition of olivine, plagioclase, and clinopyroxene components through influxes of slightly more primitive magmas, or simple crystal settling within individual dikes. These patterns may be exaggerated by the unusually low rate of re- 
Table 1. X-ray fluorescence analyses of Leg $137 / 140$ basalts and diabases.

\begin{tabular}{|c|c|c|c|c|c|c|c|c|c|c|c|c|c|c|c|}
\hline $\begin{array}{l}\text { Core, section: } \\
\text { Interval }(\mathrm{cm}) \text { : } \\
\text { Piece: } \\
\text { Depth (mbsf): } \\
\text { Lithologic unit: } \\
\text { Rock name: } \\
\text { Alteration }(\%) \text { : }\end{array}$ & $\begin{array}{c}174 \mathrm{R}-1 \\
108-110 \\
17 \\
1577.4 \\
195 \\
\text { OPC } \\
\text { n.d. }\end{array}$ & $\begin{array}{c}174 \mathrm{R}-2 \\
54-58 \\
10 \\
1578.3 \\
195 \\
\text { OPC } \\
\text { n.d. }\end{array}$ & $\begin{array}{c}177 \mathrm{R}-\mathrm{I} \\
29-32 \\
9 \\
1604.8 \\
202 \\
\text { OPC } \\
\text { n.d. }\end{array}$ & $\begin{array}{c}186 \mathrm{R}-1 \\
116-119 \\
16 \mathrm{~B} \\
1627.5 \\
213 \\
\text { A-D } \\
50\end{array}$ & $\begin{array}{c}\text { I86R-2 } \\
18-21 \\
6 \\
1628.0 \\
213 \\
\text { A-D } \\
50\end{array}$ & $\begin{array}{c}186 \mathrm{R}-2 \\
50-53 \\
10 \\
1628.3 \\
213 \\
\text { A-D } \\
50\end{array}$ & $\begin{array}{c}187 R-1 \\
5-9 \\
2 \\
1632.1 \\
214 \\
\text { A-D } \\
10\end{array}$ & $\begin{array}{c}187 R-1 \\
38-40 \\
9 \\
1632.4 \\
215 \\
\text { A-D } \\
50\end{array}$ & $\begin{array}{c}189 \mathrm{R}-1 \\
70-73 \\
16 \\
1651.7 \\
218 \\
\mathrm{M}-\mathrm{POC} \\
10\end{array}$ & $\begin{array}{c}189 \mathrm{R}-2 \\
110-113 \\
16 \\
1653.6 \\
218 \\
\mathrm{M}-\mathrm{POC} \\
70\end{array}$ & $\begin{array}{c}190 R-1 \\
5-9 \\
2 \\
1655.2 \\
218 \\
\text { M-POC } \\
70\end{array}$ & $\begin{array}{c}193 R-1 \\
17-20 \\
6 \\
1674.7 \\
220 \\
\text { M-POC } \\
50\end{array}$ & $\begin{array}{c}193 \mathrm{R}-\mathrm{I} \\
28-31 \\
9 \\
1674.8 \\
220 \\
\mathrm{M}-\mathrm{POC} \\
80\end{array}$ & $\begin{array}{c}193 \mathrm{R}-\mathrm{I} \\
44-46 \\
13 \mathrm{~A} \\
1675.0 \\
220 \\
\mathrm{M}-\mathrm{POC} \\
56\end{array}$ & $\begin{array}{c}193 R-1 \\
60-63 \\
14 \\
1675.1 \\
220 \\
\text { M-POC } \\
95\end{array}$ \\
\hline $\begin{array}{l}\mathrm{SiO}_{2}(w 1 \%) \\
\mathrm{TiO}_{2} \\
\mathrm{Al}_{2} \mathrm{O}_{3} \\
\mathrm{Fe}_{2} \mathrm{O}_{3} * \\
\mathrm{MnO}^{*} \\
\mathrm{MgO} \\
\mathrm{CaO} \\
\mathrm{Na}_{2} \mathrm{O} \\
\mathrm{K}_{2} \mathrm{O} \\
\mathrm{P}_{2} \mathrm{O}_{4} \\
\text { Total (anhyd.) }\end{array}$ & $\begin{array}{c}50.6 \\
0.90 \\
15.29 \\
10.50 \\
0.18 \\
8.16 \\
12.90 \\
1.67 \\
\text { n.d. } \\
0.06 \\
100.2\end{array}$ & $\begin{array}{c}50.5 \\
0.91 \\
15.57 \\
10.42 \\
0.18 \\
7.95 \\
12.86 \\
1.72 \\
\text { n.d. } \\
0.06 \\
100.2\end{array}$ & $\begin{array}{c}50.7 \\
1.03 \\
14.53 \\
11.33 \\
0.20 \\
8.03 \\
12.43 \\
1.81 \\
\text { n.d. } \\
0.07 \\
100.1\end{array}$ & $\begin{array}{c}47.9 \\
1.00 \\
16.08 \\
10.21 \\
0.18 \\
9.28 \\
13.46 \\
1.62 \\
\text { n.d. } \\
0.08 \\
99.8\end{array}$ & $\begin{array}{c}47.9 \\
1.04 \\
16.59 \\
9.92 \\
0.17 \\
9.34 \\
13.28 \\
1.83 \\
\text { n.d. } \\
0.08 \\
100.2\end{array}$ & $\begin{array}{c}47.1 \\
1.00 \\
16.36 \\
10.69 \\
0.18 \\
9.09 \\
13.56 \\
1.42 \\
\text { n.d. } \\
0.08 \\
99.5\end{array}$ & $\begin{array}{c}50.2 \\
1.09 \\
14.59 \\
11.16 \\
0.20 \\
8.25 \\
12.49 \\
2.13 \\
\text { n.d. } \\
0.07 \\
100.1\end{array}$ & $\begin{array}{c}51.0 \\
0.92 \\
14.46 \\
10.26 \\
0.17 \\
8.38 \\
12.77 \\
2.36 \\
\text { n.d. } \\
0.06 \\
100.3\end{array}$ & $\begin{array}{c}50.4 \\
0.94 \\
15.11 \\
10.42 \\
0.18 \\
8.32 \\
12.72 \\
1.88 \\
\text { n.d. } \\
0.07 \\
100.1\end{array}$ & $\begin{array}{c}51.1 \\
0.68 \\
15.29 \\
9.63 \\
0.17 \\
8.71 \\
12.34 \\
1.90 \\
\text { n.d. } \\
0.05 \\
99.9\end{array}$ & $\begin{array}{c}53.7 \\
0.60 \\
14.09 \\
10.07 \\
0.17 \\
8.21 \\
12.19 \\
1.13 \\
\text { n.d. } \\
0.04 \\
100.2\end{array}$ & $\begin{array}{c}50.2 \\
0.90 \\
15.02 \\
10.48 \\
0.19 \\
8.50 \\
12.50 \\
2.01 \\
\text { n.d. } \\
0.06 \\
99.9\end{array}$ & $\begin{array}{c}51.0 \\
0.46 \\
15.92 \\
8.95 \\
0.15 \\
9.22 \\
12.54 \\
2.04 \\
\text { n.d. } \\
0.03 \\
100.3\end{array}$ & $\begin{array}{c}50.5 \\
0.97 \\
15.49 \\
9.87 \\
0.18 \\
8.33 \\
13.04 \\
1.94 \\
\text { n.d. } \\
0.07 \\
100.4\end{array}$ & $\begin{array}{c}54.3 \\
0.34 \\
13.58 \\
8.66 \\
0.14 \\
7.40 \\
13.45 \\
0.80 \\
\text { n.d. } \\
0.02 \\
98.7\end{array}$ \\
\hline $\begin{array}{l}\mathrm{LOI}^{*}(\mathrm{wt} \%) \\
\mathrm{Cl}(\mathrm{ppm}) \\
\mathrm{S}\end{array}$ & $\begin{array}{l}1.62 \\
97^{1.62} \\
776\end{array}$ & $\begin{array}{l}1.56 \\
82^{789}\end{array}$ & $\begin{array}{c}1.52 \\
1023\end{array}$ & $\begin{array}{l}2.27 \\
416 \\
100\end{array}$ & $\begin{array}{l}2.54 \\
408 \\
143\end{array}$ & $\begin{array}{l}2.47 \\
436 \\
28\end{array}$ & $\begin{array}{c}1.51 \\
110^{1115}\end{array}$ & $\begin{array}{l}2.21 \\
583 \\
100\end{array}$ & $\begin{array}{l}11.58 \\
115 \\
1146\end{array}$ & $\begin{array}{l}3.44 \\
356 \\
153\end{array}$ & $\begin{array}{l}5.09 \\
283^{5} \\
364\end{array}$ & $\begin{array}{l}1.85 \\
262^{1.8} \\
438\end{array}$ & $\begin{array}{l}3.51 \\
270 \\
108\end{array}$ & $\begin{array}{l}2.15 \\
236 \\
584\end{array}$ & 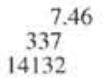 \\
\hline $\begin{array}{l}\mathrm{Nb} \\
\mathrm{Zr} \\
\mathrm{Y} \\
\mathrm{Sr} \\
\mathrm{Rb} \\
\mathrm{Ga} \\
\mathrm{Zn} \\
\mathrm{Cu} \\
\mathrm{Ni} \\
\mathrm{Cr} \\
\mathrm{V}\end{array}$ & $\begin{array}{c}<0.3 \\
42.1 \\
23.5 \\
46.6 \\
<0.5 \\
17.1 \\
70 \\
82 \\
96 \\
249 \\
298\end{array}$ & $\begin{array}{c}0.4 \\
43.6 \\
24.7 \\
46.7 \\
<0.5 \\
15.5 \\
71 \\
79 \\
95 \\
262 \\
313\end{array}$ & $\begin{array}{r}0.7 \\
46.6 \\
27.0 \\
45.8 \\
<0.5 \\
16.2 \\
83 \\
81 \\
80 \\
142 \\
335\end{array}$ & $\begin{array}{c}1.0 \\
59.5 \\
26.2 \\
64.9 \\
<0.5 \\
15.5 \\
57 \\
27 \\
131 \\
382 \\
290\end{array}$ & $\begin{array}{r}0.7 \\
61.5 \\
25.6 \\
67.5 \\
<0.5 \\
14.9 \\
58 \\
31 \\
143 \\
374 \\
285\end{array}$ & $\begin{array}{r}0.6 \\
59.2 \\
25.8 \\
63.6 \\
<0.5 \\
16.0 \\
56 \\
13 \\
137 \\
378 \\
299\end{array}$ & $\begin{array}{r}0.9 \\
55.7 \\
27.5 \\
60.6 \\
<0.5 \\
16.3 \\
87 \\
90 \\
85 \\
186 \\
339\end{array}$ & $\begin{array}{c}<0.3 \\
42.3 \\
24.4 \\
56.7 \\
<0.5 \\
14.8 \\
59 \\
12 \\
84 \\
210 \\
320\end{array}$ & $\begin{array}{c}0.7 \\
44.4 \\
24.2 \\
48.7 \\
<0.5 \\
16.0 \\
71 \\
106 \\
95 \\
273 \\
314\end{array}$ & $\begin{array}{c}0.5 \\
30.1 \\
17.7 \\
49.7 \\
<0.5 \\
12.9 \\
69 \\
10 \\
109 \\
367 \\
262\end{array}$ & $\begin{array}{c}<0.3 \\
27.1 \\
16.8 \\
27.6 \\
<0.5 \\
12.4 \\
71 \\
9 \\
98 \\
313 \\
239\end{array}$ & $\begin{array}{r}0.5 \\
40.4 \\
24.0 \\
49.1 \\
<0.5 \\
15.3 \\
73 \\
103 \\
91 \\
268 \\
320\end{array}$ & $\begin{array}{c}<0.3 \\
18.2 \\
12.7 \\
51.5 \\
<0.5 \\
12.0 \\
71 \\
9 \\
113 \\
406 \\
232\end{array}$ & $\begin{array}{r}0.6 \\
46.2 \\
25.7 \\
48.4 \\
<0.5 \\
15.0 \\
72 \\
103 \\
99 \\
291 \\
314\end{array}$ & $\begin{array}{c}<0.3 \\
14.7 \\
12.3 \\
31.3 \\
<0.5 \\
11.1 \\
53 \\
7 \\
83 \\
320 \\
156\end{array}$ \\
\hline $\begin{array}{l}\mathrm{Mg}^{\prime}-\text { value } \\
\mathrm{CaO} / \mathrm{Na}_{2} \mathrm{O} \\
\mathrm{Zr} / \mathrm{Y} \\
\mathrm{Ti} / \mathrm{Zr}\end{array}$ & $\begin{array}{c}0.631 \\
7.72 \\
1.79 \\
128\end{array}$ & $\begin{array}{c}0.627 \\
7.48 \\
1.77 \\
125\end{array}$ & $\begin{array}{c}0.609 \\
6.87 \\
1.73 \\
132\end{array}$ & $\begin{array}{c}0.667 \\
8.31 \\
2.27 \\
101\end{array}$ & $\begin{array}{c}0.675 \\
7.26 \\
2.40 \\
101\end{array}$ & $\begin{array}{c}0.652 \\
9.55 \\
2.29 \\
101\end{array}$ & $\begin{array}{c}0.619 \\
5.86 \\
2.03 \\
117\end{array}$ & $\begin{array}{l}0.643 \\
5.41 \\
1.73 \\
131\end{array}$ & $\begin{array}{c}0.637 \\
6.77 \\
1.83 \\
127\end{array}$ & $\begin{array}{c}0.666 \\
6.48 \\
1.70 \\
135\end{array}$ & $\begin{array}{c}0.642 \\
10.79 \\
1.61 \\
134\end{array}$ & $\begin{array}{c}0.641 \\
6.22 \\
1.68 \\
134\end{array}$ & $\begin{array}{r}0.694 \\
6.15 \\
1.43 \\
151\end{array}$ & $\begin{array}{r}0.650 \\
6.72 \\
1.80 \\
125\end{array}$ & $\begin{array}{c}0.653 \\
16.81 \\
1.20 \\
139\end{array}$ \\
\hline
\end{tabular}

Table 1 (continued).

\begin{tabular}{|c|c|c|c|c|c|c|c|c|c|c|c|c|c|c|c|}
\hline $\begin{array}{l}\text { Core, section: } \\
\text { Interval }(\mathrm{cm}) \text { : } \\
\text { Piece: } \\
\text { Depth (mbsf): } \\
\text { Lithologic unit: } \\
\text { Rock name: } \\
\text { Alteration }(\%):\end{array}$ & $\begin{array}{c}208 \mathrm{R}-1 \\
39-41 \\
10 \\
1778.4 \\
238 \\
\text { S-PCO } \\
10\end{array}$ & $\begin{array}{c}208 \mathrm{R}-1 \\
114-118 \\
23 \\
1779.1 \\
239 \\
\mathrm{M}-\mathrm{OPC} \\
10\end{array}$ & $\begin{array}{c}208 R-2 \\
69-72 \\
12 \\
1780.1 \\
239 \\
\text { M-OPC } \\
15\end{array}$ & $\begin{array}{c}209 R-1 \\
13-17 \\
3 \\
1787.6 \\
240 \\
\text { M-OPC } \\
15\end{array}$ & $\begin{array}{c}209 \mathrm{R}-1 \\
135-139 \\
16 \\
1788.9 \\
240 \\
\text { M-OPC } \\
50\end{array}$ & $\begin{array}{c}209 \mathrm{R}-2 \\
102-105 \\
17 \mathrm{~A} \\
1790.0 \\
240 \\
\text { M-OPC } \\
80\end{array}$ & $\begin{array}{c}211 \mathrm{R}-1 \\
83-87 \\
18 \\
1799.3 \\
241 \\
\text { S-POC } \\
15\end{array}$ & $\begin{array}{c}213 \mathrm{R}-1 \\
10-11 \\
4 \\
1812.6 \\
242 \\
\mathrm{~A}-\mathrm{B} \\
60\end{array}$ & $\begin{array}{c}213 R-1 \\
22-25 \\
8 \\
1812.7 \\
242 \\
\text { A-B } \\
60\end{array}$ & $\begin{array}{c}213 \mathrm{R}-1 \\
79-83 \\
21 \\
1813.3 \\
243 \\
\text { S-POC } \\
30\end{array}$ & $\begin{array}{c}213 \mathrm{R}-1 \\
121-123 \\
28 \mathrm{~A} \\
1813.7 \\
243 \\
\text { S-POC } \\
90\end{array}$ & $\begin{array}{c}214 \mathrm{R}-1 \\
58-61 \\
6 \\
1819.2 \\
244 \\
\mathrm{M}-\mathrm{POC} \\
90\end{array}$ & $\begin{array}{c}214 \mathrm{R}-1 \\
95-101 \\
12 \\
1819.6 \\
244 \\
\text { M-POC } \\
90\end{array}$ & $\begin{array}{c}214 \mathrm{R}-2 \\
58-64 \\
14 \\
1820.6 \\
244 \\
\mathrm{M}-\mathrm{POC} \\
90\end{array}$ & $\begin{array}{c}218 R-1 \\
17-19 \\
5 \\
1847.1 \\
247 \\
\text { A-D } \\
70\end{array}$ \\
\hline $\begin{array}{l}\mathrm{SiO}_{2}(\text { wt } \%) \\
\mathrm{TiO}_{2} \\
\mathrm{Al}_{2} \mathrm{O}_{1} \\
\mathrm{Fe}_{2} \mathrm{O}_{3} * \\
\mathrm{MnO} \\
\mathrm{MgO} \\
\mathrm{CaO} \\
\mathrm{Na}_{2} \mathrm{O} \\
\mathrm{K}_{2} \mathrm{O} \\
\mathrm{P}_{2} \mathrm{O}_{5} \\
\text { Total (anhyd.) }\end{array}$ & $\begin{array}{c}49.9 \\
0.76 \\
15.67 \\
9.31 \\
0.17 \\
9.40 \\
13.25 \\
1.71 \\
\text { n.d. } \\
0.05 \\
100.2\end{array}$ & $\begin{array}{c}50.0 \\
0.85 \\
15.92 \\
9.62 \\
0.17 \\
8.68 \\
13.13 \\
1.80 \\
\text { n.d. } \\
0.06 \\
100.2\end{array}$ & $\begin{array}{c}49.7 \\
0.82 \\
16.35 \\
9.33 \\
0.17 \\
8.65 \\
13.30 \\
1.83 \\
\text { n.d. } \\
0.06 \\
100.2\end{array}$ & $\begin{array}{c}49.9 \\
0.81 \\
15.80 \\
9.34 \\
0.17 \\
8.94 \\
13.25 \\
1.78 \\
\text { n.d. } \\
0.06 \\
100.0\end{array}$ & $\begin{array}{c}49.1 \\
0.74 \\
17.75 \\
8.36 \\
0.15 \\
8.85 \\
13.48 \\
1.74 \\
\text { n.d. } \\
0.05 \\
100.2\end{array}$ & $\begin{array}{c}52.3 \\
0.37 \\
13.57 \\
11.25 \\
0.17 \\
9.58 \\
11.28 \\
1.56 \\
\text { n.d. } \\
0.03 \\
100.1\end{array}$ & $\begin{array}{c}49.3 \\
0.80 \\
16.52 \\
9.55 \\
0.15 \\
9.08 \\
13.04 \\
1.79 \\
\text { n.d. } \\
0.06 \\
100.3\end{array}$ & $\begin{array}{c}50.8 \\
1.04 \\
14.42 \\
10.79 \\
0.17 \\
8.19 \\
12.75 \\
2.07 \\
\text { n.d. } \\
0.07 \\
100.3\end{array}$ & $\begin{array}{c}50.5 \\
1.09 \\
14.59 \\
10.81 \\
0.17 \\
8.15 \\
12.76 \\
2.06 \\
\text { n.d. } \\
0.07 \\
100.2\end{array}$ & $\begin{array}{c}49.8 \\
0.99 \\
14.92 \\
10.47 \\
0.18 \\
8.43 \\
12.81 \\
2.04 \\
\text { n.d. } \\
0.07 \\
99.7\end{array}$ & $\begin{array}{c}48.9 \\
0.50 \\
17.31 \\
8.80 \\
0.12 \\
9.57 \\
12.68 \\
2.08 \\
\text { n.d. } \\
0.03 \\
100.0\end{array}$ & $\begin{array}{c}49.1 \\
0.84 \\
16.24 \\
9.25 \\
0.16 \\
9.14 \\
13.39 \\
1.80 \\
\text { n.d. } \\
0.06 \\
99.9\end{array}$ & $\begin{array}{c}49.0 \\
0.56 \\
16.46 \\
9.72 \\
0.14 \\
9.79 \\
12.29 \\
1.79 \\
\text { n.d. } \\
0.04 \\
99.8\end{array}$ & $\begin{array}{c}49.2 \\
0.56 \\
16.88 \\
8.67 \\
0.13 \\
9.57 \\
12.97 \\
1.86 \\
\text { n.d. } \\
0.04 \\
99.9\end{array}$ & $\begin{array}{c}49.4 \\
1.06 \\
15.06 \\
10.71 \\
0.20 \\
8.61 \\
12.66 \\
2.33 \\
\text { n.d. } \\
0.07 \\
100.1\end{array}$ \\
\hline $\begin{array}{l}\mathrm{LOI}^{*}(\mathrm{wt} \%) \\
\mathrm{Cl}(\mathrm{ppm}) \\
\mathrm{S}\end{array}$ & $\begin{array}{l}1.31 \\
73^{1.31} \\
661\end{array}$ & $\begin{array}{l}1.31 \\
80^{836}\end{array}$ & $\begin{array}{c}1.34 \\
83^{777}\end{array}$ & $\begin{array}{c}1.27 \\
69^{7} \\
741\end{array}$ & $\begin{array}{l}127^{1.81} \\
571^{1}\end{array}$ & $\begin{array}{l}\quad 4.05 \\
510 \\
896\end{array}$ & $\begin{array}{l}1.73 \\
146 \\
282\end{array}$ & $\begin{array}{l}1.82 \\
169 \\
112\end{array}$ & $\begin{array}{l}1.79 \\
178^{1.79} \\
210\end{array}$ & $\begin{array}{c}1.24 \\
89^{1.24} \\
838\end{array}$ & $\begin{array}{l}2.66 \\
506 \\
62\end{array}$ & $\begin{array}{l}2.05 \\
143^{2.05} \\
591\end{array}$ & $\begin{array}{l}2.46 \\
630 \\
18\end{array}$ & $\begin{array}{l}2.05 \\
602 \\
66\end{array}$ & $\begin{array}{l}411^{2.09} \\
100\end{array}$ \\
\hline $\begin{array}{l}\mathrm{Nb} \\
\mathrm{Zr} \\
\mathrm{Y} \\
\mathrm{Sr} \\
\mathrm{Rb} \\
\mathrm{Ga} \\
\mathrm{Zn} \\
\mathrm{Cu} \\
\mathrm{Ni} \\
\mathrm{Cr} \\
\mathrm{V}\end{array}$ & $\begin{array}{c}<0.3 \\
35.0 \\
20.2 \\
48.8 \\
<0.5 \\
14.7 \\
63 \\
99 \\
141 \\
432 \\
261\end{array}$ & $\begin{array}{c}0.3 \\
42.4 \\
21.7 \\
58.8 \\
<0.5 \\
14.5 \\
60 \\
85 \\
113 \\
364 \\
260\end{array}$ & $\begin{array}{r}0.5 \\
40.4 \\
20.8 \\
59.4 \\
<0.5 \\
14.8 \\
58 \\
80 \\
120 \\
375 \\
257\end{array}$ & $\begin{array}{r}0.3 \\
39.7 \\
20.6 \\
57.9 \\
<0.5 \\
14.7 \\
63 \\
85 \\
114 \\
426 \\
256\end{array}$ & $\begin{array}{c}<0.3 \\
36.0 \\
18.0 \\
57.5 \\
<0.5 \\
14.4 \\
54 \\
158 \\
134 \\
389 \\
229\end{array}$ & $\begin{array}{c}<0.3 \\
18.0 \\
12.4 \\
48.4 \\
<0.5 \\
10.4 \\
69 \\
8 \\
130 \\
458 \\
187\end{array}$ & $\begin{array}{r}0.3 \\
38.6 \\
21.6 \\
53.9 \\
<0.5 \\
14.0 \\
53 \\
115 \\
144 \\
358 \\
247\end{array}$ & $\begin{array}{r}0.7 \\
48.6 \\
27.3 \\
54.5 \\
<0.5 \\
16.7 \\
49 \\
56 \\
74 \\
169 \\
337\end{array}$ & $\begin{array}{c}0.3 \\
48.4 \\
26.2 \\
54.4 \\
<0.5 \\
15.1 \\
50 \\
105 \\
75 \\
160 \\
347\end{array}$ & $\begin{array}{r}0.5 \\
49.4 \\
25.6 \\
54.7 \\
<0.5 \\
16.1 \\
79 \\
90 \\
95 \\
264 \\
317\end{array}$ & $\begin{array}{c}<0.3 \\
19.8 \\
14.2 \\
54.9 \\
<0.5 \\
13.8 \\
51 \\
11 \\
142 \\
409 \\
214\end{array}$ & $\begin{array}{r}0.5 \\
40.4 \\
22.7 \\
53.3 \\
<0.5 \\
16.0 \\
58 \\
194 \\
147 \\
370 \\
254\end{array}$ & $\begin{array}{c}<0.3 \\
28.0 \\
15.7 \\
51.7 \\
<0.5 \\
13.2 \\
53 \\
9 \\
164 \\
446 \\
211\end{array}$ & $\begin{array}{c}<0.3 \\
25.5 \\
15.3 \\
53.7 \\
<0.5 \\
13.2 \\
48 \\
11 \\
160 \\
477 \\
222\end{array}$ & $\begin{array}{r}0.6 \\
50.4 \\
27.0 \\
55.5 \\
<0.5 \\
16.1 \\
51 \\
44 \\
88 \\
252 \\
346\end{array}$ \\
\hline $\begin{array}{l}\mathrm{Mg}^{\prime} \text {-value } \\
\mathrm{CaO} / \mathrm{Na}_{2} \mathrm{O} \\
\mathrm{Zr} / \mathrm{Y} \\
\mathrm{Ti} / \mathrm{Zr}\end{array}$ & $\begin{array}{c}0.690 \\
7.75 \\
1.73 \\
130\end{array}$ & $\begin{array}{c}0.665 \\
7.29 \\
1.95 \\
120\end{array}$ & $\begin{array}{r}0.671 \\
7.27 \\
1.94 \\
122\end{array}$ & $\begin{array}{r}0.678 \\
7.44 \\
1.93 \\
122\end{array}$ & $\begin{array}{r}0.700 \\
7.75 \\
2.00 \\
123\end{array}$ & $\begin{array}{r}0.652 \\
7.23 \\
1.45 \\
124\end{array}$ & $\begin{array}{c}0.677 \\
7.28 \\
1.79 \\
124\end{array}$ & $\begin{array}{c}0.626 \\
6.16 \\
1.78 \\
129\end{array}$ & $\begin{array}{c}0.624 \\
6.19 \\
1.85 \\
135\end{array}$ & $\begin{array}{c}0.639 \\
6.28 \\
1.93 \\
120\end{array}$ & $\begin{array}{r}0.705 \\
6.10 \\
1.39 \\
150\end{array}$ & $\begin{array}{c}0.685 \\
7.44 \\
1.78 \\
124\end{array}$ & $\begin{array}{c}0.689 \\
6.87 \\
1.78 \\
119\end{array}$ & $\begin{array}{c}0.708 \\
6.97 \\
1.67 \\
132\end{array}$ & $\begin{array}{c}0.639 \\
5.43 \\
1.87 \\
126\end{array}$ \\
\hline
\end{tabular}

Notes: Rock name: $\mathrm{A}=$ aphyric $(\%) ; \mathrm{S}=$ sparsely phyric $(\%) ; \mathrm{M}=$ moderately phyric $(\%) ; \mathrm{D}=$ diabase; $\mathrm{B}=$ aphanitic groundmass. Phenocrysts are listed in order of abundance for each unit. $\mathrm{P}=$ plagioclase; $\mathrm{O}=$ olivine; $\mathrm{C}=$ clinopyroxene (Shipboard Scientific Party, 1992). $\mathrm{OPC}=$ phenocrysts as just described, but with no relation to relative abundance (first three samples only, from Leg 137). Alteration $\%=$ percentage of secondary minerals in the groundmass. $\mathrm{n} . \mathrm{d} .=$ not determined. 
Table 1 (continued).

\begin{tabular}{|c|c|c|c|c|c|c|c|c|c|c|c|c|c|c|c|c|}
\hline $\begin{array}{c}194 R-1 \\
5-9 \\
2 \\
1680.5 \\
220 \\
\text { M-POC } \\
\text { I5 }\end{array}$ & $\begin{array}{c}196 \mathrm{R}-1 \\
0-3 \\
\mathrm{I} \\
1696.5 \\
221 \\
\mathrm{~A} \\
10 \\
\end{array}$ & $\begin{array}{c}197 R-1 \\
92-95 \\
20 \\
1703.7 \\
222 \\
\text { M-POC } \\
15\end{array}$ & $\begin{array}{c}197 \mathrm{R}-1 \\
105-107 \\
23 \\
1703.9 \\
223 \\
\text { S-POC } \\
15\end{array}$ & $\begin{array}{c}198 R-1 \\
4-6 \\
2 \\
1712.2 \\
223 \\
\text { S-POC } \\
15\end{array}$ & $\begin{array}{c}198 R-1 \\
75-78 \\
19 \\
1713.0 \\
224 \\
\text { A-D } \\
60 \\
\end{array}$ & $\begin{array}{c}199 \mathrm{R}-1 \\
71-76 \\
17 \\
1720.1 \\
226 \\
\mathrm{M}-\mathrm{PCO} \\
15\end{array}$ & $\begin{array}{c}199 \mathrm{R}-1 \\
81-84 \\
19 \\
1720.2 \\
226 \\
\mathrm{M}-\mathrm{PCO} \\
15\end{array}$ & $\begin{array}{c}200 \mathrm{R}-1 \\
53-57 \\
12 \\
1729.1 \\
227 \\
\mathrm{M}-\mathrm{POC} \\
15\end{array}$ & $\begin{array}{c}200 \mathrm{R}-\mathrm{I} \\
86-88 \\
17 \mathrm{~A} \\
1729.5 \\
227 \\
\mathrm{M}-\mathrm{POC} \\
15\end{array}$ & $\begin{array}{c}201 \mathrm{R}-1 \\
5-9 \\
2 \\
1737.9 \\
228 \\
\text { A-D } \\
60\end{array}$ & $\begin{array}{c}203 R-1 \\
4-7 \\
2 \\
1749.1 \\
231 \\
\text { A-D } \\
15\end{array}$ & $\begin{array}{c}203 R-1 \\
19-21 \\
6 \\
1749.2 \\
231 \\
\text { A-D } \\
\text { I5 } \\
\end{array}$ & $\begin{array}{c}203 \mathrm{R}-1 \\
23-25 \\
7 \\
1749.2 \\
231 \\
\text { A-D } \\
15 \\
\end{array}$ & $\begin{array}{c}205 R-1 \\
78-79 \\
18 \\
1757.8 \\
234 \\
\text { A-B } \\
20 \\
\end{array}$ & $\begin{array}{c}205 R-1 \\
91-92 \\
22 \\
1757.9 \\
235 \\
\text { M-COP } \\
10\end{array}$ & $\begin{array}{c}207 R-1 \\
0-4 \\
\text { I } \\
1768.4 \\
236 \\
\text { A } \\
60 \\
\end{array}$ \\
\hline $\begin{array}{c}49.8 \\
0.84 \\
16.12 \\
9.56 \\
0.17 \\
8.58 \\
13.08 \\
1.85 \\
\text { n.d. } \\
0.05 \\
100.0\end{array}$ & $\begin{array}{c}50.5 \\
1.11 \\
14.47 \\
11.20 \\
0.19 \\
8.27 \\
12.29 \\
2.10 \\
\text { n.d. } \\
0.08 \\
100.2\end{array}$ & $\begin{array}{c}50.8 \\
1.02 \\
14.63 \\
11.03 \\
0.19 \\
8.14 \\
12.61 \\
1.86 \\
\text { n.d. } \\
0.07 \\
100.3\end{array}$ & $\begin{array}{c}50.7 \\
1.06 \\
14.53 \\
11.27 \\
0.20 \\
8.31 \\
12.37 \\
1.89 \\
\text { n.d. } \\
0.07 \\
100.4\end{array}$ & $\begin{array}{c}49.2 \\
0.79 \\
15.82 \\
9.77 \\
0.17 \\
9.56 \\
12.92 \\
1.68 \\
\text { n.d. } \\
0.05 \\
100.0\end{array}$ & $\begin{array}{c}50.8 \\
1.01 \\
14.65 \\
10.18 \\
0.18 \\
8.48 \\
12.87 \\
2.07 \\
\text { n.d. } \\
0.07 \\
100.3\end{array}$ & $\begin{array}{c}49.4 \\
0.78 \\
16.41 \\
9.10 \\
0.16 \\
9.69 \\
13.05 \\
1.76 \\
\text { n.d. } \\
0.05 \\
100.4\end{array}$ & $\begin{array}{c}49.4 \\
0.77 \\
16.23 \\
9.16 \\
0.16 \\
9.59 \\
13.01 \\
1.69 \\
\text { n.d. } \\
0.05 \\
100.1\end{array}$ & $\begin{array}{c}49.3 \\
0.75 \\
16.53 \\
9.09 \\
0.15 \\
9.43 \\
13.10 \\
1.75 \\
\text { n.d. } \\
0.05 \\
100.2\end{array}$ & $\begin{array}{c}49.5 \\
0.75 \\
16.51 \\
8.93 \\
0.16 \\
9.35 \\
13.20 \\
1.74 \\
\text { n.d. } \\
0.05 \\
100.2\end{array}$ & $\begin{array}{c}51.2 \\
0.73 \\
14.83 \\
10.53 \\
0.19 \\
8.69 \\
12.05 \\
2.00 \\
\text { n.d. } \\
0.05 \\
100.2\end{array}$ & $\begin{array}{c}50.2 \\
0.92 \\
15.01 \\
10.50 \\
0.18 \\
8.78 \\
13.03 \\
1.89 \\
\text { n.d. } \\
0.06 \\
100.6\end{array}$ & $\begin{array}{c}50.1 \\
0.93 \\
14.82 \\
10.35 \\
0.19 \\
8.61 \\
13.27 \\
1.96 \\
\text { n.d. } \\
0.06 \\
100.3\end{array}$ & $\begin{array}{c}49.8 \\
0.90 \\
15.15 \\
10.45 \\
0.18 \\
8.86 \\
12.87 \\
1.81 \\
\text { n.d. } \\
0.06 \\
100.0\end{array}$ & $\begin{array}{c}49.9 \\
1.10 \\
14.44 \\
11.19 \\
0.19 \\
8.15 \\
12.63 \\
2.17 \\
\text { n.d. } \\
0.07 \\
99.8\end{array}$ & $\begin{array}{r}50.1 \\
1.08 \\
14.39 \\
11.00 \\
0.19 \\
8.32 \\
12.82 \\
2.22 \\
\text { n.d. } \\
0.07 \\
100.2\end{array}$ & $\begin{array}{c}50.6 \\
1.01 \\
14.42 \\
10.70 \\
0.20 \\
8.58 \\
12.77 \\
1.96 \\
\text { n.d. } \\
0.07 \\
100.3\end{array}$ \\
\hline $\begin{array}{l}1.77 \\
158 \\
603\end{array}$ & $\begin{array}{l}113^{1.71} \\
933\end{array}$ & $\begin{array}{l}1.33 \\
843\end{array}$ & $\begin{array}{l}1.44 \\
119 \\
622\end{array}$ & $\begin{array}{c}1.57 \\
92 \\
729\end{array}$ & $\begin{array}{l}1.68 \\
543 \\
43\end{array}$ & $\begin{array}{c}1.58 \\
76 \\
838\end{array}$ & $\begin{array}{l}1.54 \\
82 \\
776\end{array}$ & $\begin{array}{c}1.50 \\
53 \\
780\end{array}$ & $\begin{array}{c}1.39 \\
58 \\
805\end{array}$ & $\begin{array}{l}1.80 \\
482 \\
21\end{array}$ & $\begin{array}{l}1.35 \\
\text { n.d. } \\
\text { n.d. }\end{array}$ & $\begin{array}{c}1.20 \\
93 \\
687\end{array}$ & $\begin{array}{l}1.18 \\
84 \\
951\end{array}$ & $\begin{array}{l}1.25 \\
122 \\
950\end{array}$ & $\begin{array}{l}1.31 \\
139 \\
426\end{array}$ & $\begin{array}{l}1.36 \\
235 \\
259\end{array}$ \\
\hline $\begin{array}{l}<0.3 \\
39.4 \\
22.2 \\
46.7 \\
<0.5 \\
15.3 \\
68 \\
91 \\
110 \\
348 \\
291\end{array}$ & $\begin{array}{c}0.6 \\
54.3 \\
29.3 \\
49.1 \\
<0.5 \\
16.1 \\
109 \\
79 \\
88 \\
172 \\
353\end{array}$ & $\begin{array}{r}0.3 \\
47.2 \\
26.8 \\
46.1 \\
<0.5 \\
16.0 \\
77 \\
82 \\
86 \\
228 \\
322\end{array}$ & $\begin{array}{l}<0.3 \\
50.1 \\
28.6 \\
43.7 \\
<0.5 \\
16.4 \\
79 \\
82 \\
91 \\
220 \\
358\end{array}$ & $\begin{array}{l}<0.3 \\
36.9 \\
21.7 \\
54.4 \\
<0.5 \\
14.2 \\
68 \\
88 \\
153 \\
371 \\
247\end{array}$ & $\begin{array}{r}0.5 \\
48.9 \\
26.0 \\
59.1 \\
<0.5 \\
15.9 \\
59 \\
13 \\
90 \\
270 \\
336\end{array}$ & $\begin{array}{c}0.3 \\
39.8 \\
19.9 \\
64.2 \\
<0.5 \\
14.6 \\
70 \\
88 \\
168 \\
432 \\
242\end{array}$ & $\begin{array}{r}0.5 \\
38.9 \\
19.3 \\
63.8 \\
<0.5 \\
13.6 \\
70 \\
84 \\
171 \\
430 \\
232\end{array}$ & $\begin{array}{l}<0.3 \\
37.9 \\
18.7 \\
67.0 \\
<0.5 \\
14.8 \\
60 \\
82 \\
170 \\
415 \\
218\end{array}$ & $\begin{array}{c}0.4 \\
39.1 \\
19.0 \\
65.4 \\
<0.5 \\
13.7 \\
64 \\
87 \\
161 \\
437 \\
232\end{array}$ & $\begin{array}{r}0.4 \\
33.4 \\
19.7 \\
60.2 \\
<0.5 \\
14.8 \\
68 \\
10 \\
93 \\
250 \\
287\end{array}$ & $\begin{array}{l}\text { n.d. } \\
\text { n.d. } \\
\text { n.d. } \\
\text { n.d. } \\
\text { n.d. } \\
\text { n.d. } \\
\text { n.d. } \\
\text { n.d. } \\
\text { n.d. } \\
\text { n.d. } \\
\text { n.d. }\end{array}$ & $\begin{array}{c}0.3 \\
43.0 \\
24.8 \\
52.6 \\
<0.5 \\
14.9 \\
77 \\
90 \\
104 \\
377 \\
310\end{array}$ & $\begin{array}{c}0.4 \\
44.1 \\
25.3 \\
55.0 \\
<0.5 \\
14.7 \\
81 \\
96 \\
107 \\
367 \\
290\end{array}$ & $\begin{array}{r}0.7 \\
55.6 \\
27.9 \\
59.9 \\
<0.5 \\
15.7 \\
84 \\
88 \\
81 \\
181 \\
336\end{array}$ & $\begin{array}{r}0.8 \\
55.1 \\
27.7 \\
60.2 \\
<0.5 \\
15.4 \\
75 \\
99 \\
81 \\
181 \\
341\end{array}$ & $\begin{array}{r}0.5 \\
49.4 \\
26.4 \\
55.2 \\
<0.5 \\
15.2 \\
59 \\
67 \\
95 \\
276 \\
341\end{array}$ \\
\hline $\begin{array}{c}0.664 \\
7.07 \\
1.77 \\
128\end{array}$ & $\begin{array}{l}0.619 \\
5.85 \\
1.85 \\
122\end{array}$ & $\begin{array}{c}0.619 \\
6.78 \\
1.76 \\
130\end{array}$ & $\begin{array}{r}0.619 \\
6.54 \\
1.75 \\
127\end{array}$ & $\begin{array}{c}0.683 \\
7.69 \\
1.70 \\
128\end{array}$ & $\begin{array}{l}0.647 \\
6.22 \\
1.88 \\
124\end{array}$ & $\begin{array}{c}0.701 \\
7.41 \\
2.00 \\
117\end{array}$ & $\begin{array}{c}0.697 \\
7.70 \\
2.02 \\
119\end{array}$ & $\begin{array}{l}0.695 \\
7.49 \\
2.03 \\
119\end{array}$ & $\begin{array}{l}0.697 \\
7.59 \\
2.06 \\
115\end{array}$ & $\begin{array}{r}0.645 \\
6.03 \\
1.70 \\
1.31\end{array}$ & $\begin{array}{l}0.648 \\
6.89 \\
\text { n.d. } \\
\text { n.d. }\end{array}$ & $\begin{array}{c}0.647 \\
6.77 \\
1.73 \\
130\end{array}$ & $\begin{array}{c}0.651 \\
7.11 \\
1.74 \\
123\end{array}$ & $\begin{array}{l}0.616 \\
5.82 \\
1.99 \\
118\end{array}$ & $\begin{aligned} & 0.625 \\
& 5.77 \\
& 1.99 \\
& 118\end{aligned}$ & $\begin{array}{r}0.638 \\
6.52 \\
1.87 \\
122\end{array}$ \\
\hline
\end{tabular}

Table 1 (continued).

\begin{tabular}{|c|c|c|c|c|c|c|c|c|c|c|c|c|c|c|c|}
\hline $\begin{array}{c}220 \mathrm{R}-1 \\
0-2 \\
1 \\
1865.5 \\
251 \\
\text { A-D } \\
30 \\
\end{array}$ & $\begin{array}{c}222 \mathrm{R}-\mathrm{I} \\
100-104 \\
19 \\
1885.6 \\
255 \\
\text { S-OCP } \\
60\end{array}$ & $\begin{array}{c}222 \mathrm{R}-\mathrm{I} \\
12 \mathrm{I}-125 \\
23 \\
1885.8 \\
256 \\
\text { A-D } \\
70\end{array}$ & $\begin{array}{c}224 \mathrm{R}-1 \\
78-82 \\
17 \\
1904.5 \\
259 \\
\text { A-D } \\
30 \\
\end{array}$ & $\begin{array}{c}225 \mathrm{R}-1 \\
13-16 \\
3 \\
1912.3 \\
259 \\
\text { A-D } \\
50\end{array}$ & $\begin{array}{c}225 \mathrm{R}-1 \\
103-106 \\
26 \\
1913.2 \\
259 \\
\text { A-D } \\
50\end{array}$ & $\begin{array}{c}226 \mathrm{R}-3 \\
18-24 \\
2 \\
1923.1 \\
260 \\
\mathrm{M}-\mathrm{COP} \\
60\end{array}$ & $\begin{array}{c}227 \mathrm{R}-2 \\
26-30 \\
5 \\
1926.1 \\
261 \\
\mathrm{M}-\mathrm{COP} \\
30\end{array}$ & $\begin{array}{c}228 \mathrm{R}-1 \\
0-5 \\
1 \\
1934.0 \\
262 \\
\mathrm{M}-\mathrm{OCP} \\
20\end{array}$ & $\begin{array}{c}230 R-1 \\
5-8 \\
\text { IB } \\
1953.1 \\
265 \\
\text { S-POC } \\
50\end{array}$ & $\begin{array}{c}232 \mathrm{R}-1 \\
4-6 \\
1 \\
1957.3 \\
265 \\
\text { S-POC } \\
5\end{array}$ & $\begin{array}{c}233 R-1 \\
4-7 \\
2 \\
1967.5 \\
266 \\
\text { A } \\
60\end{array}$ & $\begin{array}{c}235 \mathrm{R}-\mathrm{I} \\
0-3 \\
1 \\
1976.1 \\
267 \\
\mathrm{M}-\mathrm{PCO} \\
40\end{array}$ & $\begin{array}{c}235 \mathrm{R}-1 \\
18-20 \\
6 \\
1976.3 \\
268 \\
\text { S-PC } \\
40 \\
\end{array}$ & $\begin{array}{c}236 \mathrm{R}-\mathrm{I} \\
0-4 \\
1 \\
1980.7 \\
269 \\
\mathrm{M}-\mathrm{POC} \\
30\end{array}$ & $\begin{array}{c}238 \mathrm{R}-1 \\
22-25 \\
8 \\
1992.3 \\
269 \\
\text { M-POC } \\
30\end{array}$ \\
\hline $\begin{array}{c}50.9 \\
1.07 \\
14.94 \\
10.16 \\
0.18 \\
7.92 \\
13.06 \\
2.31 \\
\text { n.d. } \\
0.07 \\
100.6\end{array}$ & $\begin{array}{l}\text { n.d. } \\
\text { n.d. } \\
\text { n.d. } \\
\text { n.d. } \\
\text { n.d. } \\
\text { n.d. } \\
\text { n.d. } \\
\text { n.d. } \\
\text { n.d. } \\
\text { n.d. } \\
\text { n.d. }\end{array}$ & $\begin{array}{c}49.2 \\
0.96 \\
15.69 \\
10.21 \\
0.15 \\
9.02 \\
12.82 \\
1.67 \\
\text { n.d. } \\
0.08 \\
99.8\end{array}$ & $\begin{array}{c}50.8 \\
1.14 \\
14.17 \\
11.74 \\
0.19 \\
8.01 \\
11.69 \\
2.25 \\
\text { n.d. } \\
0.08 \\
100.1\end{array}$ & $\begin{array}{c}49.8 \\
0.98 \\
15.29 \\
9.68 \\
0.18 \\
8.81 \\
13.20 \\
1.92 \\
\text { n.d. } \\
0.06 \\
99.9\end{array}$ & $\begin{array}{c}49.7 \\
0.92 \\
15.46 \\
9.65 \\
0.17 \\
8.98 \\
13.13 \\
1.85 \\
\text { n.d. } \\
0.06 \\
99.9\end{array}$ & $\begin{array}{c}49.2 \\
0.77 \\
16.91 \\
9.34 \\
0.16 \\
9.10 \\
13.32 \\
1.76 \\
\text { n.d. } \\
0.05 \\
100.6\end{array}$ & $\begin{array}{c}50.5 \\
0.88 \\
15.72 \\
10.00 \\
0.17 \\
8.28 \\
13.09 \\
1.77 \\
\text { n.d. } \\
0.06 \\
100.5\end{array}$ & $\begin{array}{c}49.6 \\
0.82 \\
16.03 \\
9.88 \\
0.18 \\
8.85 \\
13.06 \\
1.79 \\
\text { n.d. } \\
0.06 \\
100.2\end{array}$ & $\begin{array}{c}50.9 \\
1.01 \\
14.79 \\
10.76 \\
0.18 \\
8.29 \\
12.18 \\
2.15 \\
\text { n.d. } \\
0.07 \\
100.3\end{array}$ & $\begin{array}{c}50.5 \\
0.98 \\
14.91 \\
10.83 \\
0.19 \\
8.33 \\
12.16 \\
2.28 \\
\text { n.d. } \\
0.07 \\
100.2\end{array}$ & $\begin{array}{c}50.3 \\
0.89 \\
14.85 \\
10.13 \\
0.15 \\
8.90 \\
13.24 \\
1.80 \\
\text { n.d. } \\
0.06 \\
100.3\end{array}$ & $\begin{array}{c}50.5 \\
0.92 \\
15.60 \\
9.97 \\
0.18 \\
8.48 \\
13.15 \\
1.96 \\
\text { n.d. } \\
0.07 \\
100.8\end{array}$ & $\begin{array}{c}50.8 \\
0.94 \\
14.75 \\
10.35 \\
0.15 \\
8.46 \\
12.87 \\
1.88 \\
\text { n.d. } \\
0.07 \\
100.3\end{array}$ & $\begin{array}{c}49.5 \\
0.78 \\
17.02 \\
8.82 \\
0.15 \\
8.55 \\
13.59 \\
1.85 \\
\text { n.d. } \\
0.06 \\
100.3\end{array}$ & $\begin{array}{c}50.1 \\
0.86 \\
15.65 \\
9.60 \\
0.17 \\
8.53 \\
13.00 \\
1.91 \\
\text { n.d. } \\
0.06 \\
99.9\end{array}$ \\
\hline $\begin{array}{c}1.34 \\
449 \\
39\end{array}$ & $\begin{array}{l}\text { n.d. } \\
184 \\
344\end{array}$ & $\begin{array}{l}2.67 \\
348 \\
27\end{array}$ & $\begin{array}{l}1.53 \\
538 \\
504\end{array}$ & $\begin{array}{l}1.34 \\
359 \\
187\end{array}$ & $\begin{array}{c}1.50 \\
406 \\
95\end{array}$ & $\begin{array}{l}1.74 \\
153 \\
333\end{array}$ & $\begin{array}{c}1.40 \\
49 \\
735\end{array}$ & $\begin{array}{c}1.80 \\
94^{1063}\end{array}$ & $\begin{array}{c}1.48 \\
446 \\
90\end{array}$ & $\begin{array}{c}0.69 \\
88 \\
159\end{array}$ & $\begin{array}{c}1.57 \\
647 \\
23\end{array}$ & $\begin{array}{l}1.16 \\
82 \\
826\end{array}$ & $\begin{array}{l}1.68 \\
183 \\
167\end{array}$ & $\begin{array}{l}11.52 \\
599\end{array}$ & $\begin{array}{l}1.14 \\
108 \\
795\end{array}$ \\
\hline $\begin{array}{r}0.4 \\
50.8 \\
26.8 \\
61.2 \\
<0.5 \\
16.0 \\
42 \\
26 \\
79 \\
231 \\
339\end{array}$ & $\begin{array}{l}0.3 \\
48.3 \\
25.9 \\
50.6 \\
<0.5 \\
15.7 \\
56 \\
94 \\
81 \\
187 \\
324\end{array}$ & $\begin{array}{r}0.6 \\
57.3 \\
25.1 \\
65.6 \\
<0.5 \\
15.5 \\
45 \\
10 \\
137 \\
462 \\
290\end{array}$ & $\begin{array}{r}0.5 \\
53.9 \\
30.3 \\
53.6 \\
<0.5 \\
16.6 \\
57 \\
89 \\
76 \\
127 \\
374\end{array}$ & $\begin{array}{c}<0.3 \\
56.7 \\
24.2 \\
77.3 \\
<0.5 \\
15.3 \\
40 \\
107 \\
112 \\
416 \\
278\end{array}$ & $\begin{array}{r}0.5 \\
52.1 \\
23.2 \\
77.0 \\
<0.5 \\
14.8 \\
40 \\
58 \\
115 \\
414 \\
174\end{array}$ & $\begin{array}{l}<0.3 \\
35.9 \\
20.0 \\
54.7 \\
<0.5 \\
15.2 \\
48 \\
148 \\
151 \\
363 \\
244\end{array}$ & $\begin{array}{l}<0.3 \\
43.1 \\
23.2 \\
54.0 \\
<0.5 \\
15.3 \\
76 \\
80 \\
101 \\
278 \\
279\end{array}$ & $\begin{array}{r}0.6 \\
39.0 \\
21.9 \\
52.7 \\
<0.5 \\
15.2 \\
62 \\
94 \\
133 \\
394 \\
261\end{array}$ & $\begin{array}{l}<0.3 \\
46.1 \\
26.1 \\
51.6 \\
<0.5 \\
15.0 \\
44 \\
45 \\
84 \\
178 \\
327\end{array}$ & $\begin{array}{c}<0.3 \\
47.7 \\
26.0 \\
52.5 \\
<0.5 \\
16.4 \\
90 \\
86 \\
88 \\
168 \\
347\end{array}$ & $\begin{array}{l}<0.3 \\
41.2 \\
24.5 \\
47.8 \\
<0.5 \\
15.1 \\
39 \\
20 \\
92 \\
207 \\
323\end{array}$ & $\begin{array}{c}<0.3 \\
44.7 \\
23.4 \\
60.2 \\
<0.5 \\
15.4 \\
58 \\
86 \\
97 \\
358 \\
276\end{array}$ & $\begin{array}{r}0.4 \\
44.4 \\
23.8 \\
48.9 \\
<0.5 \\
16.0 \\
42 \\
98 \\
89 \\
266 \\
324\end{array}$ & $\begin{array}{c}0.6 \\
38.9 \\
20.0 \\
61.2 \\
<0.5 \\
15.4 \\
49 \\
114 \\
125 \\
369 \\
243\end{array}$ & $\begin{array}{c}<0.3 \\
41.6 \\
22.1 \\
59.3 \\
<0.5 \\
15.9 \\
63 \\
84 \\
105 \\
377 \\
273\end{array}$ \\
\hline $\begin{array}{c}0.632 \\
5.67 \\
1.90 \\
126\end{array}$ & $\begin{array}{l}\text { n.d. } \\
\text { n.d. } \\
1.86 \\
\text { n.d. }\end{array}$ & $\begin{array}{l}0.660 \\
7.68 \\
2.28 \\
101\end{array}$ & $\begin{array}{c}0.600 \\
5.20 \\
1.78 \\
127\end{array}$ & $\begin{array}{c}0.667 \\
6.89 \\
2.34 \\
103\end{array}$ & $\begin{array}{c}0.672 \\
7.10 \\
2.25 \\
106\end{array}$ & $\begin{array}{c}0.682 \\
7.57 \\
1.80 \\
129\end{array}$ & $\begin{array}{c}0.646 \\
7.40 \\
1.86 \\
122\end{array}$ & $\begin{array}{c}0.663 \\
7.29 \\
1.78 \\
125\end{array}$ & $\begin{array}{c}0.629 \\
5.67 \\
1.77 \\
131\end{array}$ & $\begin{array}{c}0.629 \\
5.33 \\
1.83 \\
123\end{array}$ & $\begin{array}{c}0.659 \\
7.36 \\
1.68 \\
129\end{array}$ & $\begin{array}{c}0.652 \\
6.71 \\
1.91 \\
123\end{array}$ & $\begin{aligned} 0.643 \\
6.85 \\
1.87 \\
127\end{aligned}$ & $\begin{array}{c}0.681 \\
7.37 \\
1.95 \\
120\end{array}$ & $\begin{array}{c}0.662 \\
6.81 \\
1.88 \\
124\end{array}$ \\
\hline
\end{tabular}


Table 2. X-ray fluorescence analytical statistics and comparisons.

\begin{tabular}{|c|c|c|c|c|c|c|c|c|c|c|}
\hline & \multicolumn{2}{|c|}{ BIR-I } & \multicolumn{2}{|c|}{ DNC-1 } & \multicolumn{2}{|c|}{ BAS-1II } & \multirow[b]{2}{*}{ BAS- 140} & \multirow[b]{2}{*}{$\begin{array}{c}\text { Detection } \\
\text { limits }(2 \sigma)\end{array}$} & \multicolumn{2}{|c|}{ Mean difference } \\
\hline & Mean & $\begin{array}{l}\text { Std. dev. } \\
\text { (1 } \sigma)\end{array}$ & Mean & $\begin{array}{c}\text { Std. dev. } \\
(1 \sigma)\end{array}$ & Mean & $\begin{array}{l}\text { Std. dev. } \\
(I \sigma)\end{array}$ & & & $\begin{array}{l}\text { (Stanford- } \\
\text { Shipboard) }\end{array}$ & $\begin{array}{l}\text { Std. dev. } \\
(1 \sigma)\end{array}$ \\
\hline $\begin{array}{l}\mathrm{SiO}_{2}(\text { wt } \%) \\
\mathrm{TiO}_{2} \\
\mathrm{Al}_{2} \mathrm{O}_{1} \\
\mathrm{Fe}_{2} \mathrm{O}_{3} * \\
\mathrm{MnO} \\
\mathrm{MgO} \\
\mathrm{CaO} \\
\mathrm{Na}_{2} \mathrm{O} \\
\mathrm{K}_{2} \mathrm{O} \\
\mathrm{P}_{2} \mathrm{O}_{4} \\
\mathrm{LOI} \\
\text { Total }\end{array}$ & $\begin{array}{c}47.8 \\
0.96 \\
15.65 \\
11.38 \\
0.174 \\
9.65 \\
13.31 \\
1.83 \\
0.028 \\
0.022 \\
-0.75 \\
100.1\end{array}$ & $\begin{array}{l}0.2 \\
0.01 \\
0.09 \\
0.05 \\
0.003 \\
0.09 \\
0.05 \\
0.03 \\
0.002 \\
0.002\end{array}$ & $\begin{array}{c}46.9 \\
0.49 \\
18.58 \\
10.04 \\
0.151 \\
10.12 \\
11.33 \\
1.90 \\
0.233 \\
0.066 \\
-0.10 \\
99.7\end{array}$ & $\begin{array}{l}0.2 \\
0.00 \\
0.09 \\
0.07 \\
0.003 \\
0.07 \\
0.05 \\
0.04 \\
0.002 \\
0.002\end{array}$ & $\begin{array}{c}49.5 \\
0.79 \\
16.43 \\
9.04 \\
0.156 \\
8.56 \\
13.22 \\
1.82 \\
0.011 \\
0.052 \\
0.42 \\
100.0\end{array}$ & $\begin{array}{l}0.2 \\
0.01 \\
0.07 \\
0.04 \\
0.002 \\
0.07 \\
0.03 \\
0.03 \\
0.001 \\
0.002\end{array}$ & $\begin{array}{c}50.6 \\
0.99 \\
14.58 \\
11.08 \\
0.19 \\
8.14 \\
12.41 \\
1.85 \\
\text { n.d. } \\
0.065 \\
\text { n.d. } \\
99.9\end{array}$ & $\begin{array}{l}\text { n.d. } \\
\text { n.d. } \\
\text { n.d. } \\
\text { n.d. } \\
\text { n.d. } \\
\text { n.d. } \\
\text { n.d. } \\
\text { n.d. } \\
\text { n.d. } \\
\text { n.d. }\end{array}$ & $\begin{array}{c}-0.2 \\
-0.02 \\
-0.17 \\
0.10 \\
-0.002 \\
-0.21 \\
-0.03 \\
-0.01 \\
\text { n.d. } \\
0.014\end{array}$ & $\begin{array}{l}0.25 \\
0.01 \\
0.13 \\
0.15 \\
0.01 \\
0.12 \\
0.08 \\
0.06 \\
\text { n.d. } \\
0.03\end{array}$ \\
\hline $\begin{array}{l}\mathrm{Cl}(\mathrm{ppm}) \\
\mathrm{S} \\
\mathrm{Nb} \\
\mathrm{Zr} \\
\mathrm{Y} \\
\mathrm{Sr} \\
\mathrm{Rb} \\
\mathrm{Ga} \\
\mathrm{Zn} \\
\mathrm{Cu} \\
\mathrm{Ni} \\
\mathrm{Cr} \\
\mathrm{V}\end{array}$ & $\begin{array}{c}\text { n.d. } \\
\text { n.d. } \\
0.5 \\
15.6 \\
14.9 \\
108 \\
<0.5 \\
16.3 \\
73.0 \\
129 \\
159 \\
380 \\
311\end{array}$ & $\begin{array}{l}\text { n.d. } \\
\text { n.d. } \\
0.1 \\
0.4 \\
0.3 \\
1 \\
0.4 \\
0.2 \\
0.4 \\
1 \\
1 \\
2 \\
4\end{array}$ & $\begin{array}{c}60 \\
648 \\
1.3 \\
36.9 \\
16.6 \\
143 \\
3.3 \\
14.5 \\
70.6 \\
89 \\
246 \\
276 \\
149\end{array}$ & $\begin{array}{l}4 \\
2 \\
0.1 \\
0.4 \\
0.2 \\
0 \\
0.1 \\
0.4 \\
0.4 \\
1 \\
1 \\
1 \\
3\end{array}$ & $\begin{array}{c}79 \\
729 \\
0.5 \\
39.6 \\
20.1 \\
59.9 \\
<0.5 \\
14.6 \\
68.3 \\
87 \\
127 \\
370 \\
245\end{array}$ & $\begin{array}{l}3 \\
5 \\
0.1 \\
0.2 \\
0.2 \\
0.4 \\
0.2 \\
0.4 \\
0.5 \\
1 \\
1 \\
2 \\
2\end{array}$ & $\begin{array}{c}87 \\
887 \\
<0.3 \\
44.8 \\
26.6 \\
45.5 \\
<0.5 \\
15.6 \\
85 \\
84 \\
85 \\
192 \\
342\end{array}$ & $\begin{array}{l}4 \\
2 \\
0.3 \\
0.5 \\
0.5 \\
0.5 \\
0.5 \\
0.8 \\
1 \\
1 \\
1 \\
1 \\
3\end{array}$ & $\begin{array}{r}\text { n.d. } \\
\text { n.d. } \\
-0.1 \\
0.3 \\
-0.1 \\
-0.4 \\
\text { n.d. } \\
\text { n.d. } \\
-11 \\
0 \\
-1 \\
-12 \\
-2\end{array}$ & $\begin{array}{l}\text { n.d. } \\
\text { n.d. } \\
0.2 \\
0.5 \\
0.7 \\
0.8 \\
\text { n.d. } \\
\text { n.d. } \\
3 \\
2 \\
3 \\
6 \\
6\end{array}$ \\
\hline
\end{tabular}

Note: BAS-140 was ignited before major element analysis; therefore, its total is reported on an anhydrous basis.

Table 3. Barium and rare-earth element abundances, lithologic Unit 220.

\begin{tabular}{|c|c|c|c|c|c|c|c|c|c|c|}
\hline \multirow{2}{*}{$\begin{array}{l}\text { Core, section: } \\
\text { Interval }(\mathrm{cm}) \text { : } \\
\text { Piece number: } \\
\text { Depth (mbsf): }\end{array}$} & \multirow{2}{*}{$\begin{array}{c}193 R-1 \\
17-20 \\
6 \\
1674.7 \\
\end{array}$} & \multirow{2}{*}{$\begin{array}{c}193 R-1 \\
28-31 \\
9 \\
1674.8\end{array}$} & \multirow{2}{*}{$\begin{array}{c}193 \mathrm{R}-1 \\
4446 \\
13 \mathrm{~A} \\
1675.0\end{array}$} & \multirow{2}{*}{$\begin{array}{c}193 R-1 \\
60-63 \\
14 \\
1675.1\end{array}$} & \multirow{2}{*}{$\begin{array}{c}194 R-1 \\
5-9 \\
2 \\
1680.5\end{array}$} & \multirow[b]{2}{*}{$\begin{array}{l}\text { Precision } \\
\left( \pm 1 \sigma_{\text {ppm }}\right)\end{array}$} & \multicolumn{3}{|c|}{ Standards } & \multirow[b]{2}{*}{$\mathrm{N}$} \\
\hline & & & & & & & BIR-1 & DNC-1 & NBS-688 & \\
\hline $\mathrm{Ba}(\mathrm{ppm})$ & 1.0 & 1.5 & 1.4 & 0.7 & 0.7 & 0.25 & 21 & 102 & 180 & - \\
\hline La & 0.86 & 0.76 & 0.92 & 0.30 & 0.77 & 0.03 & 0.77 & 3.60 & 4.98 & 0.33 \\
\hline $\mathrm{Ce}$ & 3.48 & 1.67 & 3.92 & 1.50 & 3.20 & 0.10 & 2.59 & 8.65 & 12.35 & 0.88 \\
\hline Pr & 0.73 & 0.33 & 0.81 & 0.33 & 0.65 & 0.01 & 0.46 & 1.12 & 1.78 & 0.112 \\
\hline $\mathrm{Nd}$ & 4.10 & 2.09 & 4.82 & 2.25 & 4.16 & 0.09 & 2.78 & 5.18 & 8.28 & 0.60 \\
\hline $\mathrm{Sm}$ & 1.75 & 0.88 & 1.87 & 0.92 & 1.67 & 0.06 & 1.31 & 1.57 & 2.40 & 0.181 \\
\hline Eu & 0.71 & 0.44 & 0.74 & 0.67 & 0.65 & 0.04 & 0.58 & 0.67 & 0.99 & 0.069 \\
\hline $\mathrm{Gd}$ & 2.50 & 1.44 & 2.94 & 1.45 & 2.49 & 0.05 & 2.11 & 2.18 & 2.90 & 0.249 \\
\hline $\mathrm{Tb}$ & 0.53 & 0.30 & 0.60 & 0.29 & 0.48 & 0.02 & 0.47 & 0.43 & 0.50 & 0.047 \\
\hline Dy & 3.51 & 2.20 & 4.04 & 1.96 & 3.39 & 0.07 & 3.03 & 2.83 & 3.25 & 0.322 \\
\hline Ho & 0.81 & 0.47 & 0.90 & 0.42 & 0.74 & 0.02 & 0.74 & 0.70 & 0.71 & 0.070 \\
\hline $\mathrm{Er}$ & 2.44 & 1.52 & 2.68 & 1.31 & 2.26 & 0.12 & 2.08 & 2.10 & 2.03 & 0.200 \\
\hline $\mathrm{Tm}$ & 0.35 & 0.20 & 0.40 & 0.18 & 0.33 & 0.01 & 0.30 & 0.33 & 0.31 & 0.030 \\
\hline $\mathrm{Yb}$ & 2.41 & 1.44 & 2.63 & 1.18 & 2.06 & 0.08 & 2.24 & 2.06 & 2.01 & 0.200 \\
\hline $\mathrm{Lu}$ & 0.33 & 0.21 & 0.37 & 0.16 & 0.31 & 0.01 & 0.33 & 0.30 & 0.31 & 0.034 \\
\hline
\end{tabular}

Notes: Precision = calculated precision $(\sigma)$ for Unit 220 (see text). Chondrite-normalizing value $(N)$ for Dy is from Boynton (1989); all others from Haskin et al. (1968).

covery around $1550 \mathrm{mbsf}$, due to numerous problems with coring during Legs 111, 137, and 140 (e.g., Shipboard Scientific Party, 1992).

A significant trend of $\mathrm{Zn}$ depletion with depth (Fig. 8A) occurs in the bottom $350 \mathrm{~m}$ of core, and is observed in all samples ranging from $5 \%$ to $95 \%$ alteration (Shipboard Scientific Party, 1992; this study). With the exception of the sulfide mineralization zone (910-928 mbsf) and other transition zonebasalts, typical $\mathrm{Zn}$ concentrations for 504B volcanics and sheeted dikes vary between 60 and $80 \mathrm{ppm}$. However, beginning at about $1650 \mathrm{mbsf}$, the mean $\mathrm{Zn}$ concentration $(\approx 70 \mathrm{ppm})$ decreases to about $42 \mathrm{ppm}$ at $2000 \mathrm{mbsf}$. Similarly, the minimum $\mathrm{Zn}$ abundance decreases (55-30 ppm) through the same depth interval. The relative $\mathrm{Zn}$ depletion as depicted in Figure $8 \mathrm{~A}$, is not as pronounced as the one reported by the Leg 140 Scientific Party. The difference is that the $\mathrm{Zn}$ data produced by the shipboard XRF lab, although precise, was found to be consistently $12 \mathrm{ppm}$ lower than those determined by this study and from previous legs, and has been corrected for a more precise comparison. The resulting depletion pattern is different in one significant aspect only - the depth at which the trend begins is very likely between 1700 and $1800 \mathrm{mbsf}$, and not higher up in the sheeted dike section $(\approx 1300 \mathrm{mbsf})$, as it may have appeared before (i.e., Shipboard Scientific Party, 1992; Dick et al., 1992). A comparable depletion trend for $\mathrm{Mn}$ or $\mathrm{Cu}$ has not been identified (Fig. 8B, -C). Below $1600 \mathrm{mbsf}$, the pattern of $\mathrm{MnO}$ concentrations is similar to that of $\mathrm{Fe}_{2} \mathrm{O}_{3}{ }_{3}^{*}$, and $\mathrm{Cu}$ is highly scattered around typical values of 75 to $110 \mathrm{ppm}$.

\section{VARIABILITY OF “IMMOBILE” ELEMENTS TI, ZR, AND Y}

As previously noted, all samples recovered from Leg 140 have undergone minor to extensive levels of subsolidus alteration (Shipboard Scientific Party, 1992), as indicated by mineralogy (5\%-95\% secondary minerals), water content $\left(\mathrm{LOI}^{*}=0.7 \%-7.5 \%\right)$, and concentrations of relatively mobile elements $(\mathrm{Cu}=7-194 \mathrm{ppm} ; \mathrm{S}=18$ $14132 \mathrm{ppm} ; \mathrm{Sr}=28-77 \mathrm{ppm} ;$ and $\left.\mathrm{Na}_{2} \mathrm{O}=0.8 \%-2.4 \%\right)$. The extent of 
Table 4. Comparison of samples with variable phenocryst content.

\begin{tabular}{|c|c|c|c|c|c|c|c|c|c|c|c|c|}
\hline & \multicolumn{4}{|c|}{$\begin{array}{c}\text { Aphyric } \\
(\leq 1 \% \text { phenocrysts })\end{array}$} & \multicolumn{4}{|c|}{$\begin{array}{c}\text { Sparsely phyric } \\
(1 \%-2 \% \text { phenocrysts })\end{array}$} & \multicolumn{4}{|c|}{$\begin{array}{c}\text { Moderately phyric } \\
(2 \%-10 \% \text { phenocrysts })\end{array}$} \\
\hline & Mean & 1 std. dev. & Min. & Max. & Mean & I std. dev. & Min. & Max. & Mean & I std. dev. & Min. & Max. \\
\hline $\mathrm{SiO}_{2}(\mathrm{wt} \%)$ & 50.0 & 1.1 & 47.1 & 51.2 & 50.2 & 0.7 & 49.2 & 50.9 & 49.9 & 0.5 & 49.1 & 50.7 \\
\hline $\mathrm{TiO}_{2}$ & 0.99 & 0.10 & 0.73 & 1.14 & 0.93 & 0.12 & 0.76 & 1.06 & 0.86 & 0.09 & 0.74 & 1.08 \\
\hline $\mathrm{Al}_{2} \mathrm{O}_{3}$ & 14.98 & 0.67 & 14.17 & 16.59 & 15.17 & 0.68 & 14.53 & 16.52 & 15.91 & 0.80 & 14.39 & 17.75 \\
\hline $\mathrm{Fe}_{2} \mathrm{O}_{3} *$ & 10.52 & 0.54 & 9.65 & 11.74 & 10.37 & 0.69 & 9.31 & 11.27 & 9.73 & 0.74 & 8.36 & 11.33 \\
\hline $\mathrm{MnO}$ & 0.18 & 0.01 & 0.15 & 0.20 & 0.17 & 0.02 & 0.15 & 0.20 & 0.17 & 0.01 & 0.15 & 0.20 \\
\hline $\mathrm{MgO}$ & 8.59 & 0.42 & 7.92 & 9.34 & 8.67 & 0.53 & 8.14 & 9.56 & 8.69 & 0.49 & 7.95 & 9.69 \\
\hline $\mathrm{CaO}$ & 12.86 & 0.47 & 11.69 & 13.56 & 12.69 & 0.38 & 12.16 & 13.25 & 13.05 & 0.28 & 12.43 & 13.59 \\
\hline $\mathrm{Na}_{2} \mathrm{O}$ & 1.98 & 0.23 & 1.42 & 2.36 & 1.92 & 0.20 & 1.68 & 2.28 & 1.83 & 0.12 & 1.67 & 2.22 \\
\hline $\mathrm{K}, \mathrm{O}$ & n.d. & n.d. & n.d. & n.d. & n.d. & n.d. & n.d. & n.d. & n.d. & n.d. & n.d. & n.d. \\
\hline $\mathrm{P}_{2} \mathrm{O}_{5}$ & 0.07 & 0.01 & 0.05 & 0.08 & 0.06 & 0.01 & 0.05 & 0.07 & 0.06 & 0.01 & 0.05 & 0.07 \\
\hline $\mathrm{LOI}^{*}(\mathrm{wt} \%)$ & 1.67 & 0.41 & 1.18 & 2.54 & 1.39 & 0.31 & 0.69 & 1.73 & 1.54 & 0.25 & 1.14 & 2.15 \\
\hline $\mathrm{Cl}(\mathrm{ppm})$ & 335 & 188 & 84 & 647 & 150 & 112 & 73 & 446 & 109 & 55 & 49 & 262 \\
\hline $\mathrm{S}$ & 342 & 383 & 21 & 1115 & 474 & 295 & 90 & 843 & 739 & 200 & 333 & 1146 \\
\hline $\mathrm{Nb}$ & 0.5 & 0.2 & 0.3 & 1.0 & 0.3 & 0.1 & 0.3 & 0.5 & 0.4 & 0.2 & 0.3 & 0.8 \\
\hline $\mathrm{Zr}$ & 50.5 & 7.3 & 33.4 & 61.5 & 44.4 & 5.5 & 35.0 & 50.1 & 41.6 & 4.2 & 35.9 & 55.1 \\
\hline $\mathrm{Y}$ & 25.9 & 2.3 & 19.7 & 30.3 & 24.6 & 2.7 & 20.2 & 28.6 & 22.2 & 2.7 & 18.0 & 27.7 \\
\hline $\mathrm{Sr}$ & 59.5 & 8.0 & 47.8 & 77.3 & 50.5 & 3.7 & 43.7 & 54.7 & 55.8 & 6.8 & 45.8 & 67.0 \\
\hline $\mathrm{Rb}$ & $<0.5$ & n.d. & n.d. & n.d. & $<0.5$ & n.d. & n.d. & n.d. & $<0.5$ & n.d. & n.d. & n.d. \\
\hline $\mathrm{Ga}$ & 15.5 & 0.7 & 14.7 & 16.7 & 15.5 & 0.9 & 14.0 & 16.4 & 15.2 & 0.8 & 13.6 & 17.1 \\
\hline $\mathrm{Zn}$ & 62 & 19 & 39 & 109 & 65 & 16 & 42 & 90 & 65 & 9 & 48 & 83 \\
\hline $\mathrm{Cu}$ & 57 & 36 & 10 & 107 & 88 & 18 & 45 & 115 & 95 & 21 & 79 & 158 \\
\hline $\mathrm{Ni}$ & 98 & 21 & 74 & 143 & 105 & 29 & 81 & 153 & 119 & 29 & 80 & 171 \\
\hline $\mathrm{Cr}$ & 271 & 98 & 127 & 416 & 267 & 91 & 168 & 432 & 337 & 83 & 142 & 437 \\
\hline $\mathrm{V}$ & 314 & 43 & 174 & 374 & 308 & 41 & 247 & 358 & 274 & 37 & 218 & 341 \\
\hline $\mathrm{Mg}^{\prime}$-value & 0.64 & 0.02 & 0.60 & 0.67 & 0.65 & 0.03 & 0.62 & 0.69 & 0.66 & 0.03 & 0.61 & 0.70 \\
\hline $\mathrm{CaO} / \mathrm{Na} \cdot \mathrm{O}$ & 6.61 & 1.03 & 5.20 & 9.55 & 6.69 & 0.84 & 5.33 & 7.75 & 7.17 & 0.51 & 5.77 & 7.75 \\
\hline $\mathrm{Zr} / \mathrm{Y}$ & 1.95 & 0.24 & 1.68 & 2.40 & 1.80 & 0.07 & 1.70 & 1.93 & 1.88 & 0.11 & 1.68 & 2.06 \\
\hline $\mathrm{Ti} / \mathrm{Zr}$ & 120 & 12 & 101 & 135 & 127 & 4 & 120 & 131 & 123 & 5 & 115 & 134 \\
\hline
\end{tabular}

this alteration is extremely heterogeneous on a scale of centimeters to meters, commonly ranging from slight, to nearly complete recrystallization within $10 \mathrm{~cm}$ of core. Localized areas of extensive alteration, dubbed alteration "patches" or "halos," typically represent $70 \%$ to 90\% replacement of primary minerals by actinolite, chlorite, albite, titanite, and trace epidote, prehnite, and anhydrite, whereas, sulfide minerals are virtually absent (Alt et al., 1989b; Shipboard Scientific Party, 1992). For detailed descriptions and compositions of alteration minerals in the sheeted dike complex, see Alt et al. (1985, 1986a, 1989b), Ishizuka (1989), Kempton et al. (1985), Kusakabe et al. (1989), and various authors, this volume. At high degrees of alteration $(>70 \%)$, elements that are normally considered to be relatively immobile during hydrothermal alteration (i.e., $\mathrm{TiO}_{2}, \mathrm{Zr}, \mathrm{Y}$ ) become highly variable, and exhibit even greater levels of depletion than has previously been observed at this site (as noted earlier, the degree of alteration is simply the total percentage of secondary mineralization - predominantly chlorite and actinolite-as determined by either thin-section point counts or visual inspection of sliced core samples). Comparing $\mathrm{TiO}_{2}, \mathrm{Zr}$, and the ratio $\mathrm{Zr} / \mathrm{Y}$ with highly mobile $\mathrm{Cu}$, illustrates this point (Figs. 9-11). For samples with less than $50 \%$ alteration of the groundmass, $\mathrm{Cu}$ is typically between 75 and $115 \mathrm{ppm}$, with minimal variation in $\mathrm{TiO}_{2}(0.9 \% \pm 0.1 \%), \mathrm{Zr}(43 \pm 5 \mathrm{ppm})$, and $\mathrm{Zr} / \mathrm{Y}(1.9 \pm 0.1)$. With increased alteration $\mathrm{Cu}$ becomes highly variable, with the addition or removal of $\mathrm{Cu}-\mathrm{Fe}$ sulfides, ranging from 10 to $158 \mathrm{ppm}$ at $50 \%$ to $60 \%$ alteration, and 7 to $194 \mathrm{ppm}$ at greater than $60 \%$ alteration. In contrast, the values of $\mathrm{TiO}_{2}, \mathrm{Zr}$, and $\mathrm{Zr} / \mathrm{Y}$ in samples that contain up to $60 \%$ secondary minerals, are more variable, but they are well within typical ranges for Hole 504B. At greater levels of alteration, however, even these elements exhibit marked variation, particularly toward depletion $\left(\mathrm{TiO}_{2}=1.1 \%-0.3 \% ; \mathrm{Zr}=66-15\right.$ $\mathrm{ppm} ; \mathrm{Zr} / \mathrm{Y}=2.6-1.2$ ). For this reason, an arbitrary limit of $60 \%$ groundmass alteration is used by this study to filter out "altered" samples when describing variations in igneous chemistry. Alternatively, a minimum limit of 35 to $60 \mathrm{ppm} \mathrm{Cu}$ could be used to achieve the same purpose. Under this scheme, however, some of the units with $\mathrm{Zr} / \mathrm{Y}$ ratios greater than 2.2 may be unduly omitted.

The largest $\mathrm{TiO}_{2}, \mathrm{Zr}$, and $\mathrm{Y}$ anomalies were recorded in lithologic Unit 220, a unit in which the average groundmass alteration is ap- proximately $10 \%$, but in localized patches reaches $60 \%$ to $95 \%$ (Shipboard Scientific Party, 1992). The composition of these patches relative to less altered samples in the unit is shown in Figure 12. Three samples (140-504B-193R-1, 17-20 cm; -193R-1, $44-46 \mathrm{~cm}$; and $-194 \mathrm{R}-1,5-9 \mathrm{~cm})$ represent slightly to moderately altered $\left(\mathrm{LOI}^{*}\right.$ $=1.9 \% \pm 0.2 \% ; \mathrm{Cu}=99 \pm 7 \mathrm{ppm})$ sections of Unit 220 . These samples were averaged and used as a "fresh" composition for normalization against two highly altered samples (140-504B-193R-1, 28-31 cm; and $-193 \mathrm{R}-1,60-63 \mathrm{~cm}$ ). Mobile elements in the highly altered samples are exceedingly variable and/or depleted with respect to the slight to moderately altered samples $\left(\mathrm{Na}_{2} \mathrm{O}=0.4-1.1\right.$ times that of the average "fresh" composition; $\mathrm{S}=0.2-26^{\prime} ; \mathrm{Cu}=0.1^{\prime} ; \mathrm{Sr}=0.7-1.1^{\prime}$ ). Concentrations of $\mathrm{TiO}_{2}, \mathrm{Zr}, \mathrm{Y}, \mathrm{P}$, and most rare-earth elements, on the other hand, are all depleted the same amount-roughly one-half those observed in the fresher samples-resulting in $\mathrm{Zr} / \mathrm{Y}$ and $\mathrm{Ti} / \mathrm{Zr}$ variations that are more limited than the overall changes in abundance. $\mathrm{Zr}$ is more depleted than $\mathrm{Ti}, \mathrm{Y}$, and the middle to heavy rareearth elements in the altered samples, producing a small degree of decoupling. La and Eu exhibit behavior slightly different from the rest of the rare-earth elements (Figs. 12 and 13). Both altered samples have small, positive Eu anomalies $\left(\mathrm{Eu} / \mathrm{Eu}^{*}<2\right)$, and one sample (140-504B-193R-1, 28-31 cm) displays an unusual enrichment in La relative to the trend of other light REEs. Positive Eu anomalies could be attributable to the presence of secondary albite (Humphris et al., 1978), or the interaction with seawater (e.g., Michard et al., 1983). However, the relative level of $\mathrm{Na}$ abundance in the two altered samples is the opposite of what would be expected if Eu was indicative of albite concentration-the sample with the strongest Eu anomaly contains the least $\mathrm{Na}$. It is not known if the REE pattern for Sample 140-504B-193R-1, 28-31 cm, represents a La enrichment, or a negative $\mathrm{Ce}$ anomaly associated with the interaction of seawater. If it is genuine La enrichment, the light rare-earth pattern differs from the patterns associated with palagonitization of glassy volcanic pillows (e.g., Ludden and Thompson, 1979; Alt and Emmermann, 1985). In these cases, the light rare-earth elements from $\mathrm{La}$ to $\mathrm{Sm}$ are all enriched relative to the fresh, glassy interiors, in contrast to the singular increase in $\mathrm{La}$ as seen here. 
Table 5. Hole 504B interleg comparisons-aphyric and sparsely phyric $(0 \%-2 \%)$ samples only.

\begin{tabular}{|c|c|c|c|c|c|c|c|c|c|c|c|c|}
\hline & \multicolumn{4}{|c|}{ Legs $69 / 70$} & \multicolumn{4}{|c|}{ Leg 83} & \multicolumn{4}{|c|}{ Leg 111} \\
\hline & Mean & I std. dev. & Min. & Max. & Mean & 1 std. dev. & Min. & Max. & Mean & 1 std. dev. & Min. & Max. \\
\hline $\mathrm{SiO}_{2}(\%)$ & 50.3 & 0.3 & 49.8 & 50.7 & 50.1 & 0.5 & 48.8 & 50.8 & 50.3 & 0.6 & 48.9 & 51.1 \\
\hline $\mathrm{TiO}_{2}$ & 0.94 & 0.04 & 0.88 & 1.00 & 1.00 & 0.08 & $\begin{array}{l}7+8.0 \\
0.84\end{array}$ & 1.14 & 0.96 & 0.12 & 0.75 & 1.24 \\
\hline $\mathrm{Al}_{2} \mathrm{O}_{4}$ & 15.61 & 0.56 & 14.90 & 16.70 & 14.83 & 0.38 & 14.31 & 16.08 & 15.23 & 0.85 & 13.80 & 16.90 \\
\hline $\mathrm{Fe}_{2} \mathrm{O}_{2}{ }^{*}$ & 9.97 & 0.56 & 8.83 & 10.69 & 10.68 & 0.60 & 9.67 & 11.62 & 10.46 & 0.84 & 9.26 & 12.50 \\
\hline MnO & 0.18 & 0.02 & 0.16 & 0.23 & 0.21 & 0.02 & 0.17 & 0.26 & 0.18 & 0.01 & 0.15 & 0.21 \\
\hline $\mathrm{MgO}$ & 8.28 & 0.18 & 7.99 & 8.55 & 8.49 & 0.37 & 7.72 & 9.09 & 8.33 & 0.41 & 7.16 & 9.18 \\
\hline $\mathrm{CaO}$ & 12.68 & 0.24 & 12.17 & 12.98 & $\begin{array}{l}8.49 \\
12.86\end{array}$ & 0.25 & 12.25 & 13.18 & 12.72 & 0.40 & 11.70 & 13.17 \\
\hline $\mathrm{Na}_{2} \mathrm{O}$ & 1.99 & 0.13 & 1.80 & 2.20 & 1.99 & 0.19 & 1.68 & 2.44 & 1.90 & 0.16 & 1.59 & 2.22 \\
\hline $\begin{array}{ll}\mathrm{K} \\
\mathrm{K}\end{array}$ & 0.14 & 0.09 & 0.04 & 0.36 & 0.02 & $<0.01$ & $<0.01$ & 0.03 & 0.01 & $<0.01$ & $<0.01$ & 0.02 \\
\hline $\mathrm{P}_{2} \mathrm{O}_{3}$ & 0.08 & 0.01 & 0.06 & 0.10 & 0.09 & 0.01 & 0.08 & 0.11 & 0.06 & 0.01 & 0.05 & 0.08 \\
\hline $\mathrm{Nb}(\mathrm{ppm})$ & 0.5 & 0.1 & 0.4 & 0.7 & 0.7 & 0.2 & 0.4 & 1.1 & 0.8 & 0.3 & 0.3 & 1.5 \\
\hline $\mathrm{Zr}$ & 47.5 & 3.0 & 43.0 & 53.0 & 50.6 & 5.5 & 38.0 & 59.0 & $\begin{array}{l}0.0 \\
48.3\end{array}$ & 5.9 & 38.4 & 64.4 \\
\hline Y & 23.3 & 1.4 & 21.5 & 25.6 & 24.7 & 2.2 & 20.5 & 28.0 & 24.5 & 2.6 & 20.1 & 30.4 \\
\hline Sr & 66.9 & 6.0 & 57.0 & 78.0 & 53.4 & 6.1 & 43.0 & 66.0 & 57.9 & 6.4 & 43.7 & 66.4 \\
\hline $\mathrm{Rb}$ & 2.2 & 2.0 & 0.4 & 7.3 & 0.5 & 0.1 & 0.4 & 0.9 & 0.1 & 0.1 & 0.0 & 0.4 \\
\hline $\mathrm{Ga}$ & 16.0 & 0.6 & 15.2 & 16.8 & 15.4 & 0.5 & 14.5 & 16.4 & 15.8 & 0.9 & 14.1 & 18.4 \\
\hline $\mathrm{Zn}$ & 78 & 7 & 66 & 90 & 85 & 11 & 67 & 104 & 74 & 10 & 55 & 99 \\
\hline $\mathrm{Cu}$ & n.d. & n.d. & n.d. & n.d. & n.d. & n.d. & n.d. & n.d. & n.d. & n.d. & n.d. & n.d. \\
\hline $\mathrm{Ni}$ & 106 & 19 & 76 & 144 & 93 & 7 & 76 & 102 & 104 & 28 & 55 & 164 \\
\hline $\mathrm{Cr}$ & 362 & 45 & 282 & 463 & 274 & 59 & 168 & 355 & 252 & 106 & 26 & 388 \\
\hline $\mathrm{v}$ & 279 & 28 & 231 & 347 & 292 & 24 & 245 & 326 & 271 & 36 & 213 & 345 \\
\hline $\mathrm{Mg}^{\prime}$-value & 0.647 & 0.012 & 0.628 & 0.671 & 0.636 & 0.021 & 0.594 & 0.666 & 0.637 & 0.029 & 0.558 & 0.686 \\
\hline $\mathrm{CaO} / \mathrm{Na}_{2} \mathrm{O}$ & 6.39 & 0.43 & 5.66 & 7.09 & 6.54 & 0.61 & 5.02 & 7.73 & 6.75 & 0.74 & 5.27 & 8.21 \\
\hline $\mathrm{Zr} / \mathrm{Y}$ & 2.04 & 0.09 & 1.90 & 2.18 & 2.06 & 0.26 & 1.60 & 2.84 & 1.97 & 0.10 & 1.78 & 2.12 \\
\hline
\end{tabular}

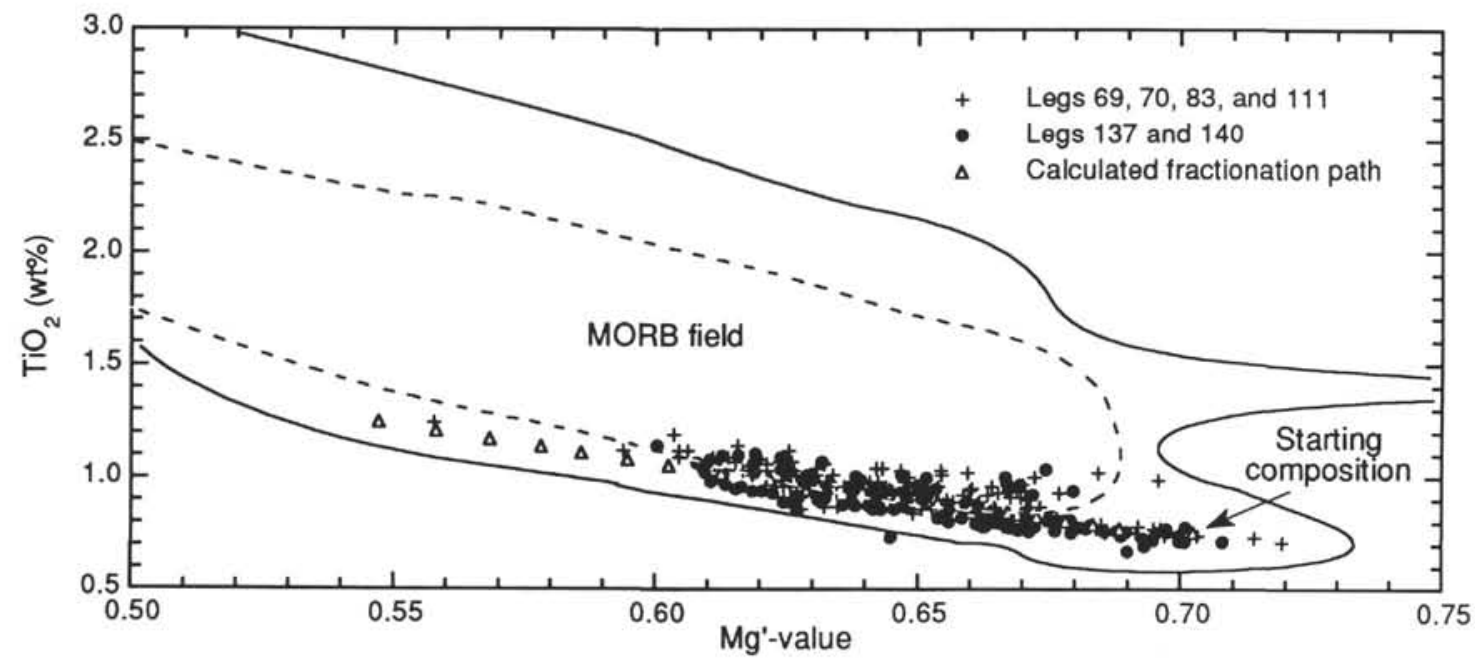

Figure 1. $\mathrm{Mg}^{\prime}$-value vs. $\mathrm{TiO}_{2}$. Compared with over 1300 aphyric to sparsely phyric MORB compositions, the Group D basalts and diabases of Hole 504B ( $>270$ analyses) are unusually low in $\mathrm{TiO}_{2}$ for a given $\mathrm{Mg}^{\prime}$-value. The calculated liquid line of descent (e.g., Nielsen, 1985, 1990) represents 38\% fractionation from the starting composition, and does not result in more "normal" MORB compositions after extensive differentiation. MORB field constructed after Autio et al. (1989). Dashed field boundary represents $95 \%$ of the MORB samples. Data from Legs 137 and 140 exclude highly (>60\%) altered samples. Analytical error is approximately the width of each plot symbol.

\section{DISCUSSION}

\section{Primary Igneous Compositions}

Discounting the mobile elements $\mathrm{K}, \mathrm{Rb}, \mathrm{Sr}, \mathrm{Cu}, \mathrm{Mn}$, and $\mathrm{Zn}$, the 127 basalts and diabases examined in this study and by the Leg 140 Shipboard Scientific Party (1992) are compositionally indistinguishable from $98 \%$ of the lithologic units observed in the upper sheeted dikes, transition zone, and volcanics of Hole 504B. Based on $\mathrm{Sr}$ and $\mathrm{Nd}$ isotopic composition (Barrett, 1983; Kusakabe et al., 1989; Shimizu et al., 1989), petrography (e.g., Natland et al., 1983; Kempton et al., 1985; Shipboard Scientific Party, 1992), and compatible element concentrations, Hole 504B Group D basalts and diabases are similar to moderately evolved, Type I MORB. On further inspection, however, Group D samples exhibit unusual levels of depletion in in- compatible elements (Figs. 1-4, and 13) and are not related to normal Type I compositions through simple fractionation. Both calculated and experimentally determined 1 -atm fractionation paths are inconsistent with the notion that normal Type I compositions can be produced through extensive fractionation of the depleted CRRZ magmas (Autio and Rhodes, 1983; Kempton et al., 1985; Autio et al., 1989, this study). The range of aphyric to sparsely $(0 \%-2 \%)$ phyric compositions observed at this location, from the most primitive to the most evolved $\left(\mathrm{Mg}^{\prime}\right.$-values $\left.=0.70-0.56\right)$, requires approximately $35 \%$ fractionation to expiain, based on calculated levels of highly incompatible elements (Autio et al., 1989) and calculated liquid lines of descent from major element phase equilibria (e.g., Nielsen, 1985, 1990). Further differentiation will produce incompatible element levels that are typical of Type I basalts, but only at the expense of reducing the compatible elements to unacceptably low levels. 
Table 5 (continued).

\begin{tabular}{cccc}
\hline \multicolumn{4}{c}{ Legs $137 / 140$} \\
\hline Mean & I std. dev. & Min. & Max. \\
\hline 50.0 & 0.6 & 48.5 & 50.8 \\
0.94 & 0.12 & 0.71 & 1.14 \\
15.01 & 0.63 & 14.17 & 16.52 \\
10.49 & 0.68 & 8.99 & 11.74 \\
0.18 & 0.01 & 0.15 & 0.20 \\
8.52 & 0.47 & 7.79 & 9.57 \\
12.79 & 0.34 & 11.69 & 13.27 \\
1.93 & 0.16 & 1.67 & 2.28 \\
0.006 & 0.005 & $<0.002$ & 0.020 \\
0.065 & 0.017 & 0.036 & 0.128 \\
0.4 & 0.2 & 0.3 & 1.5 \\
46.7 & 6.1 & 35.0 & 57.5 \\
25.0 & 2.5 & 20.2 & 30.3 \\
53.4 & 7.8 & 43.0 & 77.3 \\
$<0.5$ & 0.1 & $<0.5$ & 0.9 \\
15.6 & 0.8 & 14.0 & 16.7 \\
71 & 15 & 42 & 109 \\
93 & 17 & 56 & 136 \\
102 & 23 & 74 & 155 \\
280 & 86 & 127 & 432 \\
305 & 36 & 231 & 374 \\
0.643 & 0.027 & 0.600 & 0.701 \\
6.83 & 0.67 & 5.20 & 8.15 \\
1.80 & 0.05 & 1.64 & 1.88 \\
\hline & & & \\
\hline & & &
\end{tabular}

The $\mathrm{CaO} / \mathrm{Na}_{2} \mathrm{O}$ ratios (5.2-9.6) for Group D basalts are larger than what is commonly observed in normal MORB. These ratios are calculated to be in equilibrium with unusually anorthitic plagioclase (up to $\mathrm{An}_{92}$; Autio and Rhodes, 1983), and are in agreement with natural plagioclase compositions from the volcanic section (up to $\mathrm{An}_{90}$; Natland et al., 1983) and the upper sheeted dikes (Kempton et al., 1985). Natland et al. (1983) have suggested that the highly depleted, Ca-rich CRRZ basalts represent unusual primitive melt compositions, but this notion is inconsistent with observed concentrations of compatible elements such as $\mathrm{Ni}(102 \pm 23 \mathrm{ppm}$ in aphyric-sparsely phyric samples), and the multisaturated nature of the CRRZ basalts. Other workers have argued that these compositions are the result of a multistage melting of a normal MORB mantle source, followed by extensive crystallization (Autio and Rhodes, 1983; Autio, 1984; Kempton et al., 1985; Autio et al., 1989; Kusakabe et al., 1989; Shimizu, 1989). Multistage melting produces melts that are extremely depleted in in- compatible elements ( $\mathrm{Nb}$ and $\mathrm{Ba} \leq 1 \mathrm{ppm})$, whereas compatible elements, isotopic ratios (e.g., $\mathrm{Sr}$ and $\mathrm{Nd}$ ), and incompatible element ratios with the more incompatible element in the denominator (e.g., $\mathrm{Zr} / \mathrm{Nb}$ ) remain at normal, or slightly elevated levels (e.g., Duncan and Green, 1980) —all features that are characteristic of Group D compositions. Furthermore, multistage melting would raise $\mathrm{CaO} / \mathrm{Na}_{2} \mathrm{O}$ ratios relative to normal MORB (Green et al., 1979; Duncan and Green, 1980; Autio and Rhodes, 1983), and this increase would be reflected in higher than normal anorthite content in equilibrium plagioclase.

It is possible that multistage melting is more common than previously thought. Many mid-ocean-ridge basalts contain plagioclase phenocrysts that are too calcic to be in equilibrium with their host glasses (Bryan et al., 1976; Rhodes et al., 1979; Fisk et al., 1982). A multistage melting scenario could explain their presence, if small amounts of depleted second-stage melts were mixed with large volume, early melts during the normal production of mid-ocean-ridge basalts. The resulting mixture would erase the depleted signature of the second-stage melts, but highly calcic phenocrysts would remain (Autio et al., 1989).

The mechanism(s) that produced and erupted CRRZ melts with so little Type I MORB component is still unclear. Autio (1984) has suggested that early-stage melts could have been produced and removed from a normal MORB source at the East Pacific Rise (EPR) or the Galapagos thermal anomaly, but the difficulties of explaining the transport of the residual mantle source and the fate of the enriched, early melts are unresolved. Furthermore, little is known about the geographic extent of these Group D basalts. Among the various DSDP sites along the Cocos-Nazca Spreading Center, only basalts from Sites 501 and 505 (200 and $120 \mathrm{~km}$ south of the CRRZ, respectively; e.g., Etoubleau et al., 1983), and Site 425 (62 km north of the Galapagos Rift; e.g., Joron et al., 1980; Mattey and Muir, 1980; Srivastava et al., 1980) exhibit Group D characteristics. Basalts from DSDP Site 424, approximately $20 \mathrm{~km}$ south of the Galapagos Rift, are clearly normal Type I MORB and represent a significant compositional shift within $82 \mathrm{~km}$ of Site 425 .

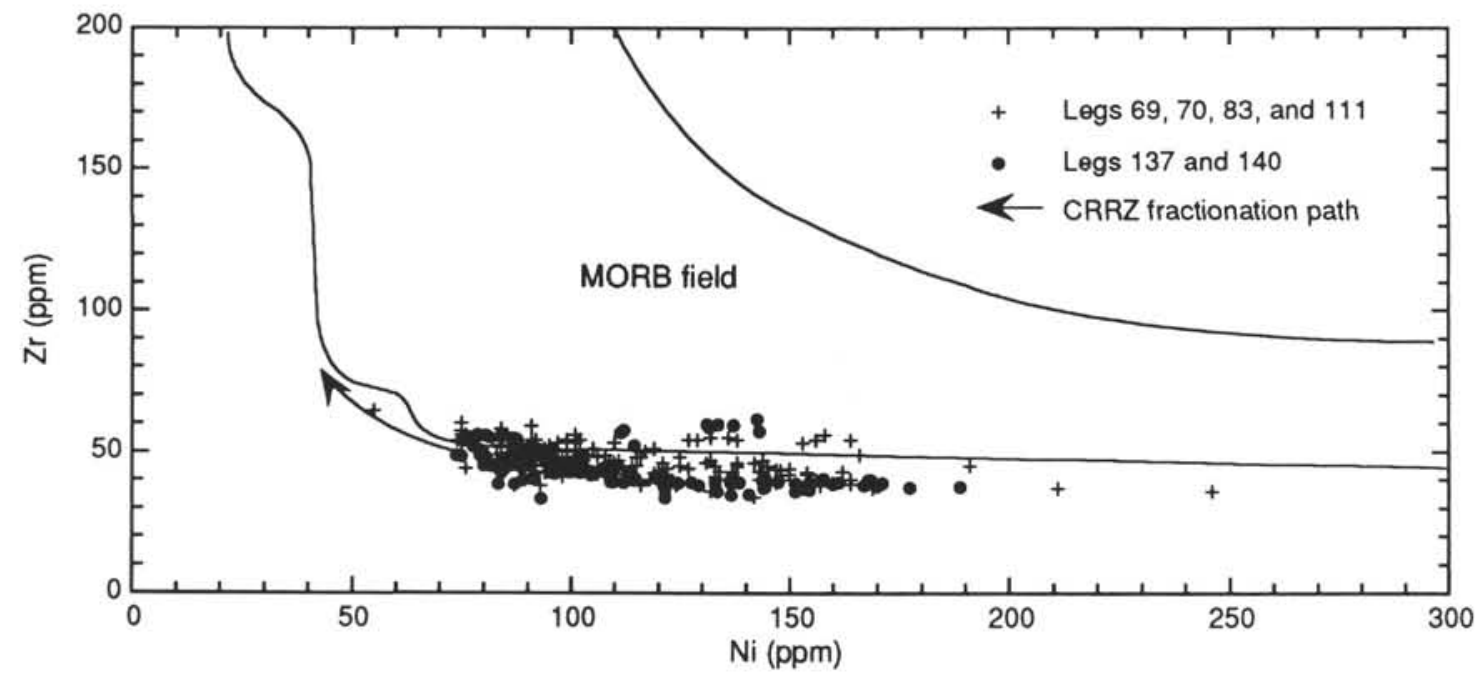

Figure 2. Ni vs. Zr. Group D compositions from Hole 504B exhibit low to moderate levels of Ni, but $\mathrm{Zr}$ values that are atypical of most MORB. The liquid line of descent calculated from 1-atm melting experiments of CRRZ basalts (Autio, 1984) demonstrates that common multiphase fractionation of CRRZ liquids will not produce normal levels of $\mathrm{Zr}$ without severely depleting Ni. MORB field constructed as in Figure 1. Data from Legs 137 and 140 exclude highly $(>60 \%$ ) altered samples. Analytical error is within the width of each plot symbol. 


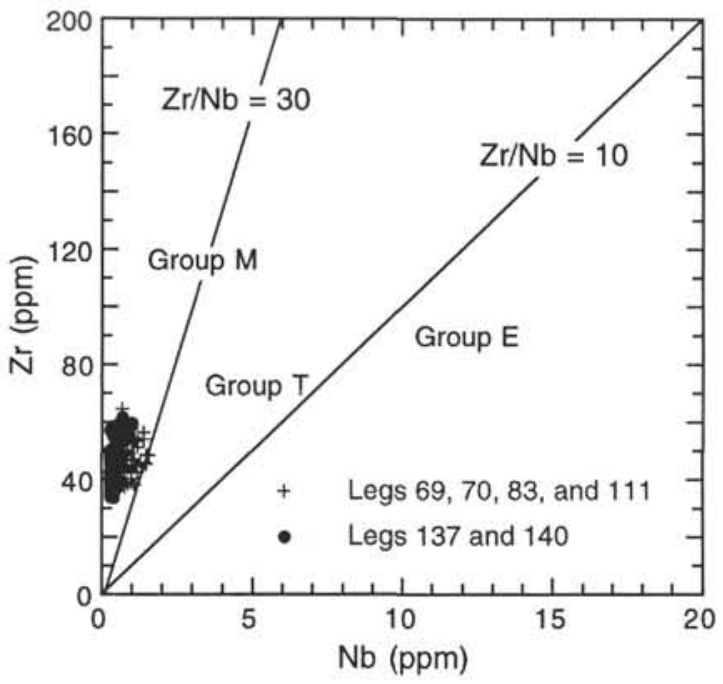

Figure 3. Nb vs. Zr. All samples from this study plot in the D Group classification of Autio and Rhodes (1983), bringing the total number of samples in this class to over 270 . Although the $\mathrm{Zr} / \mathrm{Nb}$ ratios are similar to Group $\mathrm{M}(\geq$ 30 ), or normal Type 1 MORB, the Group D compositions are extremely depleted in $\mathrm{Nb}$ and $\mathrm{Zr}$. Group E (Type II: Enriched) and Type T (Transitional) are the other basalt classes observed at Hole 504B. Data from Legs 137 and 140 exclude highly $(>60 \%)$ altered samples. Analytical error is approximately the width of each plot symbol.

\section{Variations in Ti, Zr, Y, and REEs in Hydrothermally Altered Samples}

The localized zones of $\mathrm{Ti}, \mathrm{Zr}, \mathrm{Y}, \mathrm{REE}, \mathrm{Cu}$, and $\mathrm{S}$ depletion occur in areas of enhanced fluid flow and porosity in the halos around veins and vugs (Alt et al., 1992; this study). The nature of these vugs is unclear. It has been suggested that they represent primary pore space, subsequently filled with secondary minerals (Scientific Shipboard

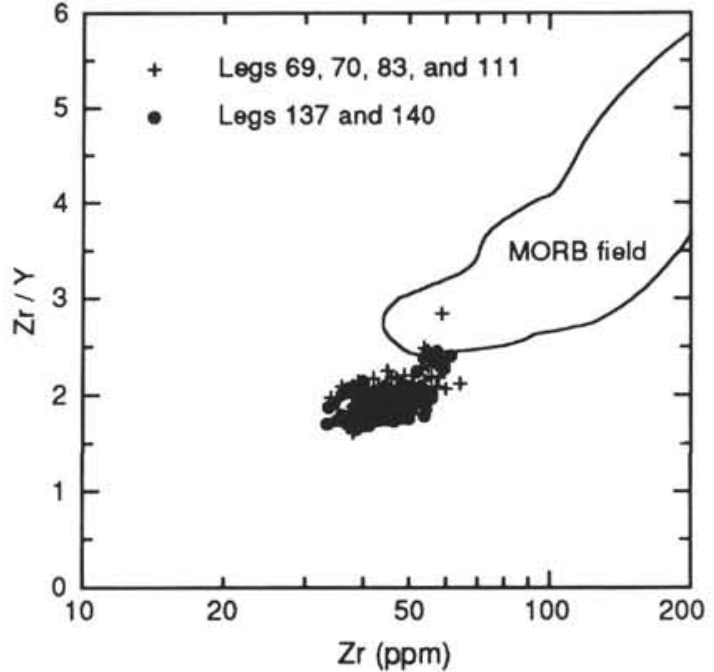

Figure 4. $\mathrm{Zr}$ vs. $\mathrm{Zr} / \mathrm{Y}$. The depleted samples of Hole 504B have $\mathrm{Zr} / \mathrm{Y}$ ratios and $\mathrm{Zr}$ concentrations that are lower than typical MORB values. MORB field plotted as in Figure 1. Data from Legs 137 and 140 exclude highly $(>60 \%)$ altered samples. Analytical error is within the width of each plot symbol.

Party, 1992), but the amount of primary porosity is likely to be vanishingly small, given the depth and pressure of magma emplacement (e.g., Moore, 1979). An alternative explanation that the vugs are the result of complete recrystallization of localized regions in glassy or fine-grained groundmass (Scientific Shipboard Party, 1992) is more likely, as glass reacts more readily than the crystalline material between $70^{\circ}$ and $300^{\circ} \mathrm{C}$ (Seyfried and Bischoff, 1979, 1981). The depletion of these elements is not restricted to the lower sheeted dike complex. Alt and Emmermann (1985) reported $\mathrm{TiO}_{2}$ loss in hydrothermally altered samples from Leg 83 , which penetrated the lower volcanic section, transition zone, and upper sheeted dike complex. Furthermore, data from lithologic Unit 60 (Emmermann, 1985) sug-
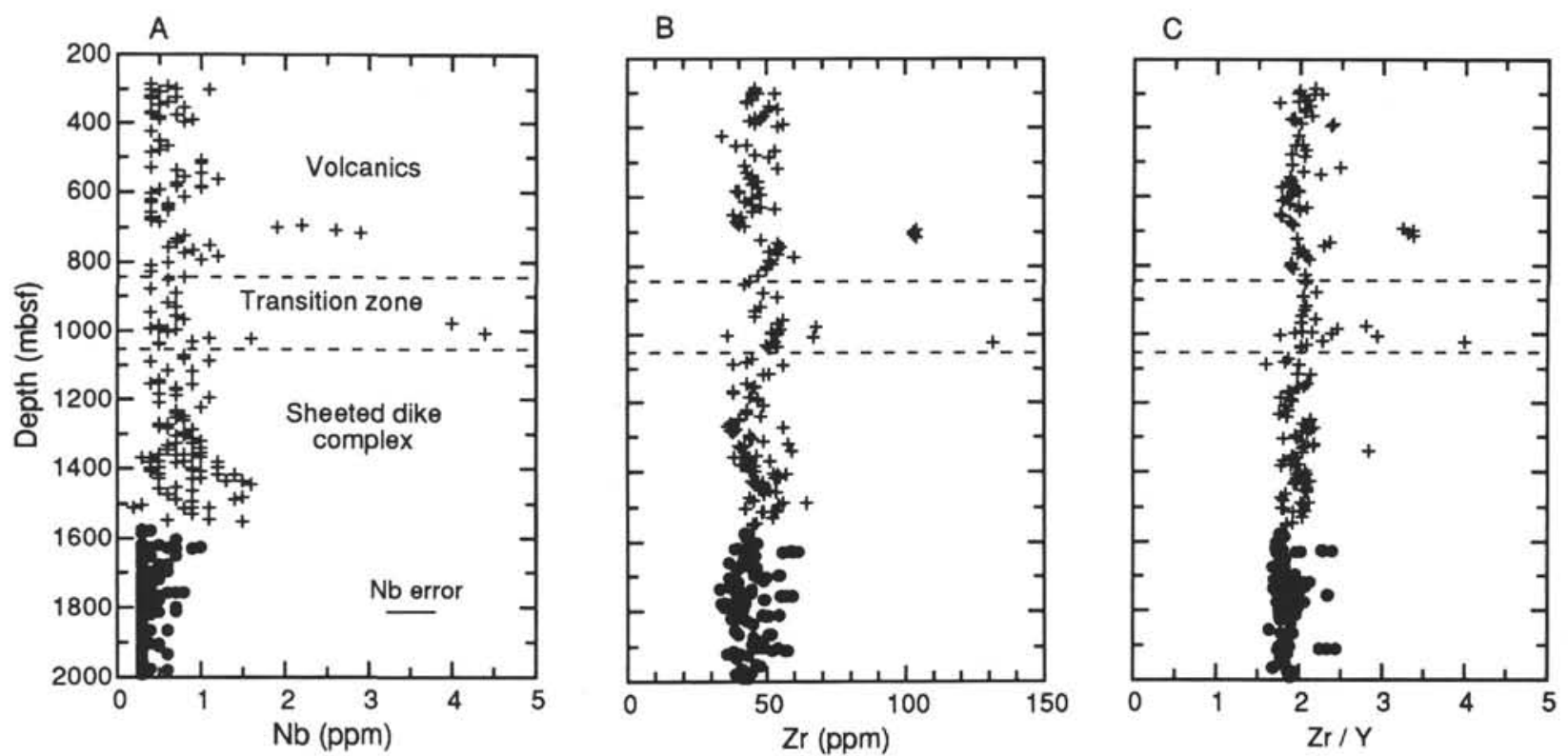

Figure 5. Depth vs. $\mathrm{Nb}(\mathbf{A}), \mathrm{Zr}(\mathbf{B}), \mathrm{Zr} / \mathrm{Y}$ (C). All samples, aphyric to moderately phyric $(\leq 10 \%)$, with less than $60 \%$ alteration are plotted. Crosses $=$ data from Legs 69, 70, 83, and 111; solid circles = data from Legs 137 and 140. Except for Nb, analytical error is within the width of each plot symbol. The error bar for $\mathrm{Nb}$ is given in Figure $5 \mathrm{~A}$. 


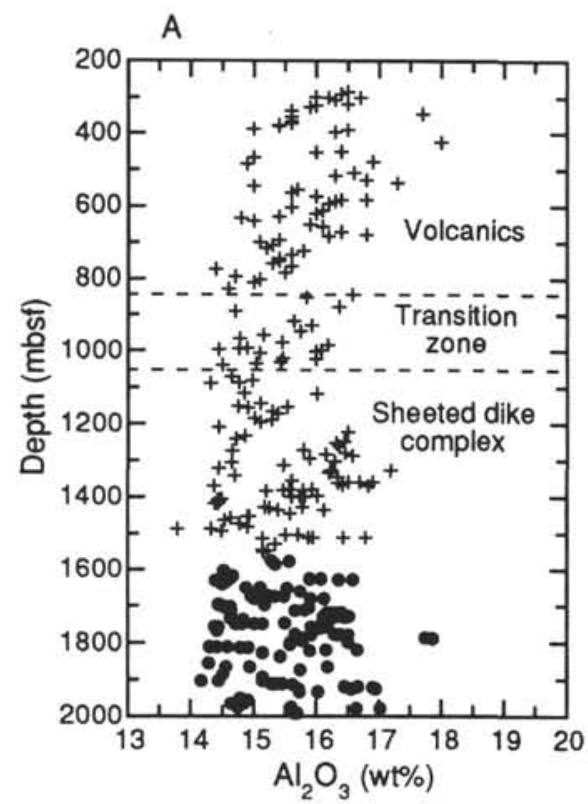

B

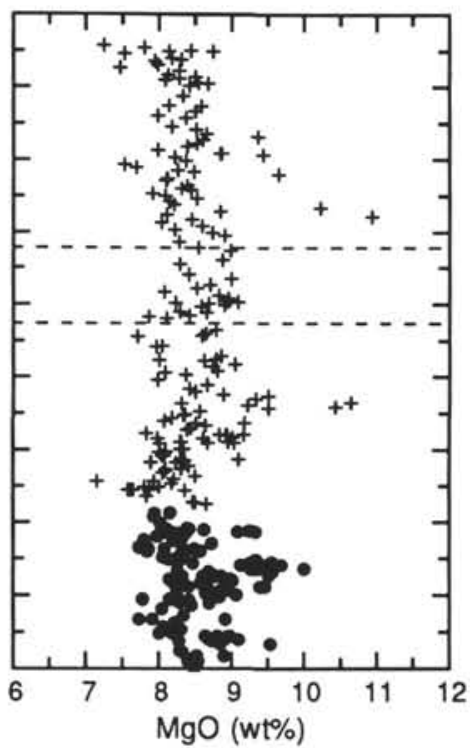

C

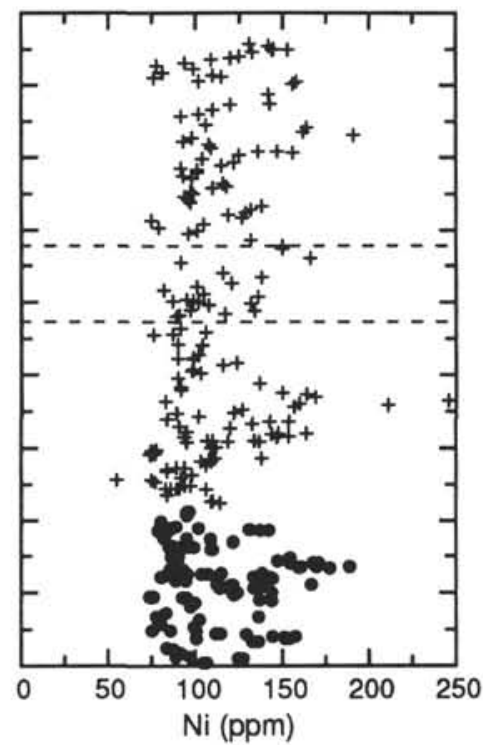

Figure 6. Depth vs. $\mathrm{Al}_{2} \mathrm{O}_{3}(\mathbf{A}), \mathrm{MgO}(\mathbf{B})$. $\mathrm{Ni}(\mathbf{C})$. All samples, aphyric to moderately phyric $(\leq 10 \%)$, with less than $60 \%$ alteration are plotted. Symbols and analytical error are the same as in Figure 5.
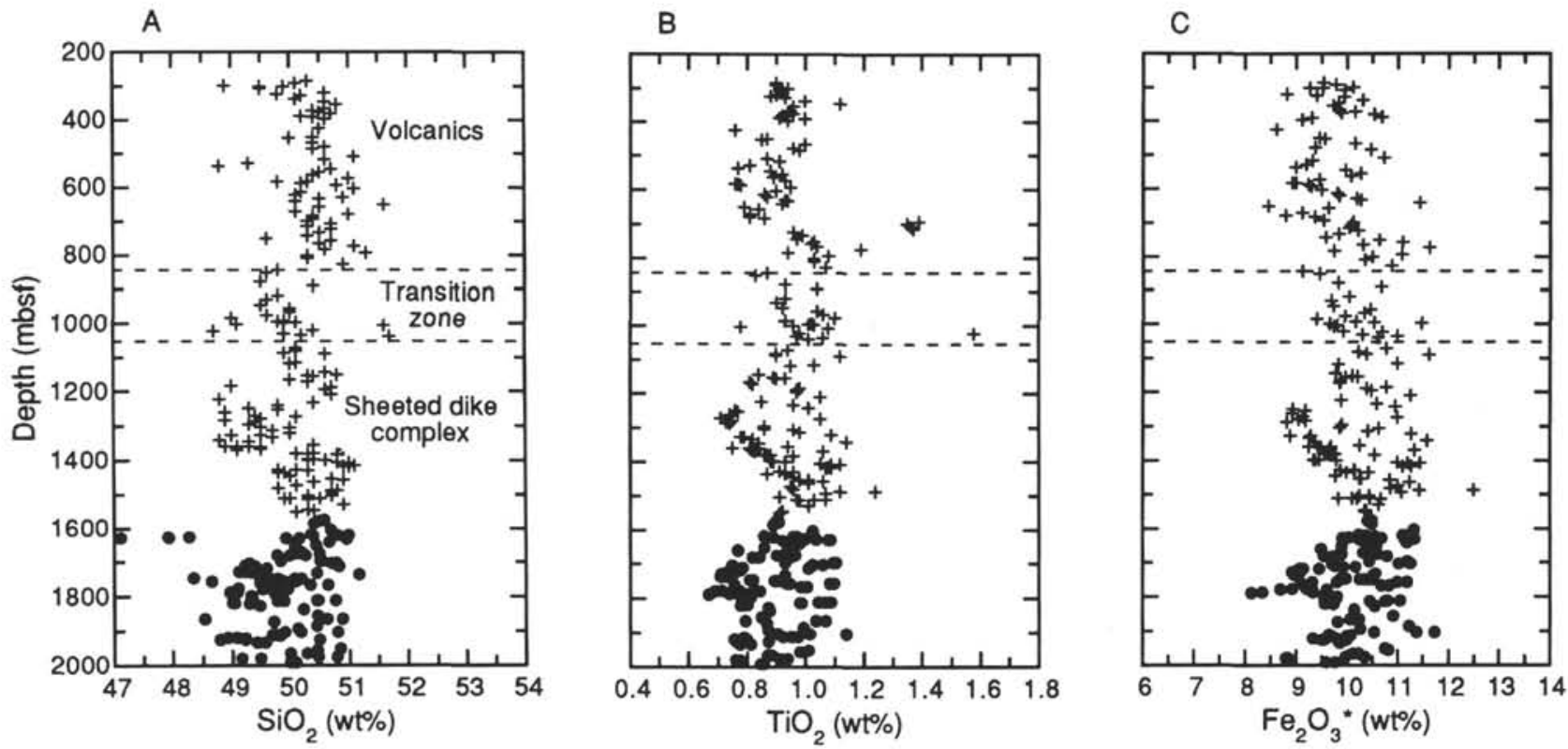

Figure 7. Depth vs. $\mathrm{SiO}_{2}(\mathbf{A}), \mathrm{TiO}_{2}(\mathbf{B}), \mathrm{Fe}_{2} \mathrm{O}_{3} *$ (C). All samples, aphyric to moderately phyric ( $\left.\leq 10 \%\right)$, with less than $60 \%$ alteration are plotted. Symbols and analytical error are the same as in Figure 5.

gests the loss of $\mathrm{Zr}(61-38 \mathrm{ppm}), \mathrm{Y}(27-17 \mathrm{ppm}), \mathrm{Cu}(118-15 \mathrm{ppm})$, and rare-earth elements as well.

Documenting the variability of $\mathrm{Ti}, \mathrm{Y}, \mathrm{Zr}$, and REE is made easier by the fact that igneous trends involving these elements are reasonably well defined for this location (Figs. 1 and 2). Comparing relatively fresh samples with the highly altered patches or halos, the most noticeable variation is a trend toward moderately strong depletion $(30 \%-50 \%)$ that is echoed by many of the rare-earth elements and $\mathrm{P}_{2} \mathrm{O}_{5}$ (Figs. 12 and 13). The abundances of these elements in the alteration patches are significantly lower than all other values reported at this site and, as such, they are easily distinguishable from relatively fresh material. Examples of alteration-induced enrichment, on the other hand, are more difficult to substantiate given that all samples lie within the normal range of primary $\mathrm{Ti}, \mathrm{Y}$, and $\mathrm{Zr}$ variation. Most of the enriched samples, although moderately recrystallized, do not contain intense alteration patches, nor do they display significant differences in secondary mineralogy (i.e., increased titanite or $\mathrm{Fe}-\mathrm{Ti}$ oxides). As a result, it is not currently known if the enrichments are a primary signature, or an artifact of alteration.

The overall effect of the alteration zones is to significantly increase the compositional scatter of moderate to highly incompatible elements (Fig. 14). Employing an alteration "filter" such as $\mathrm{H}_{2} \mathrm{O}^{+}$ 


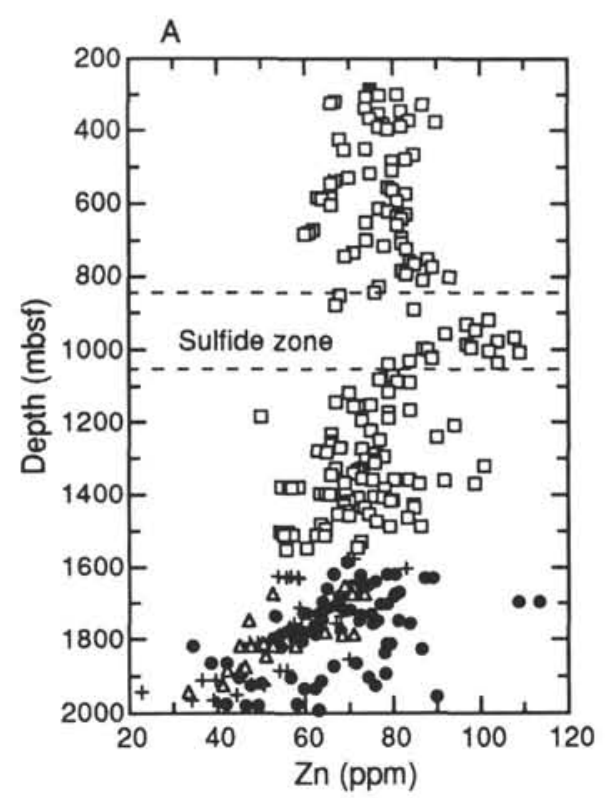

B

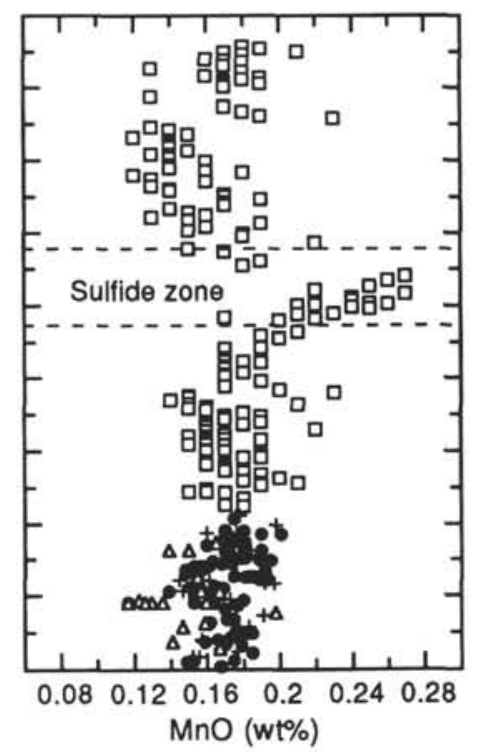

C

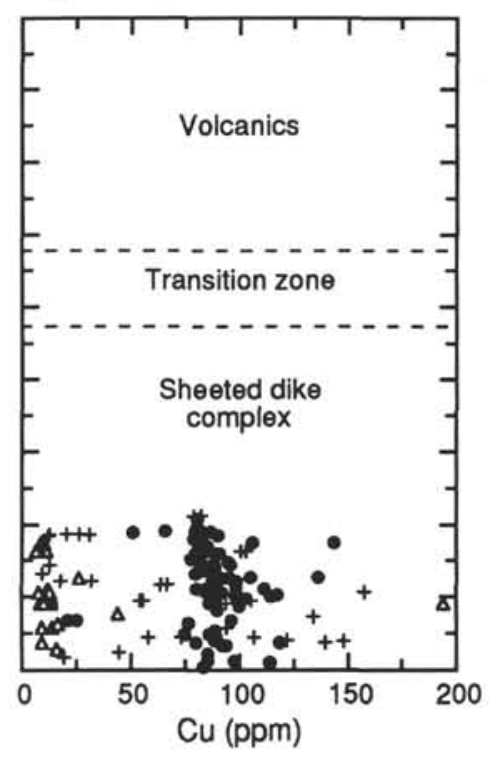

Figure 8. Depth vs. $\mathrm{Zn}(\mathbf{A}), \mathrm{MnO}$ (B), Cu (C). All samples, regardless of the degree of alteration, are plotted. Open boxes $=$ data from Legs 69, 70, 83, and 111 ; solid circles = data from Legs 137 and 140 with less than $50 \%$ alteration; crosses $=$ data from Legs 137 and 140 with $50 \%-60 \%$ alteration; open triangles $=$ data from Legs 137 and 140 with greater than $60 \%$ alteration. Analytical error is within the width of each plot symbol.

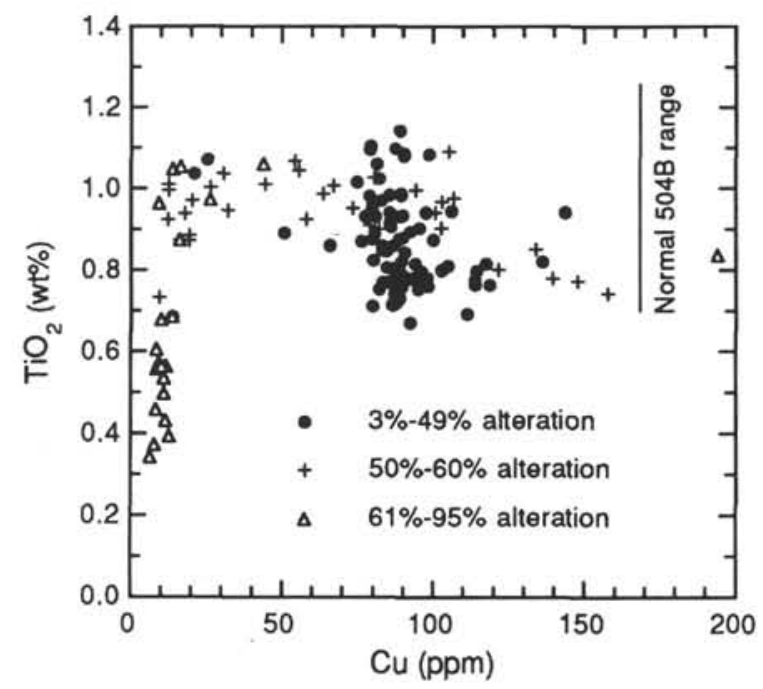

Figure 9. Cu vs. $\mathrm{TiO}_{2}$. Most Leg $137 / 140$ samples with up to $60 \%$ alteration fall within the normal range of Hole 504B variation, as defined by analyses of slightly altered samples in the upper levels of the hole. Samples with over $60 \%$ alteration display abnormally low levels of $\mathrm{TiO}_{2}$. In the absence of petrographic data, highly mobile Cu could possibly be used as an alteration indicator. The amount of error in estimating alteration is roughly $\pm 10 \%$.

(e.g., Shipboard Scientific Party, 1992) or Cu content to separate the affected samples is useful, but inexact. The arbitrary definition of $\leq$ $60 \%$ secondary minerals as being "unaltered" in terms of $\mathrm{Ti}, \mathrm{Zr}$, and $\mathrm{Y}$ has been used in this study, but this method is inexact as well, and may be too permissive if future work reveals the enriched samples to be the result of alteration.

Rare-earth elements in hydrothermal fluids from mid-ocean-ridge vents are enriched 10 to 10,000 times relative to seawater (Michard et al., 1983; Campbell et al., 1988), and it is intriguing to think that the intense alteration zones could be one of the main sources of $\mathrm{Y}$ and

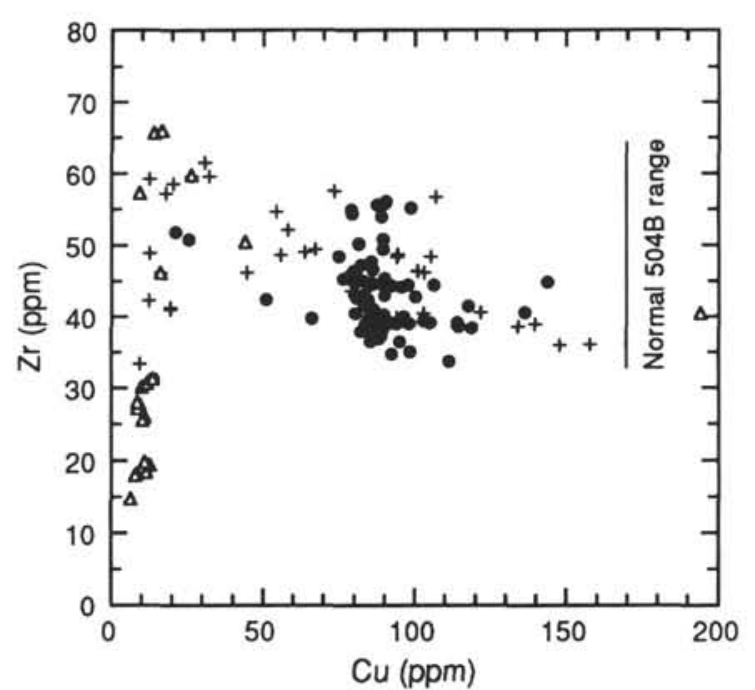

Figure 10. Cu vs. Zr. As in Figure 9, the Zr concentrations in most Leg 137/ 140 samples up to $60 \%$ alteration are within the typical Hole 504B range. At more intense levels of alteration, $\mathrm{Zr}$ is commonly lower.

REE enrichment. This notion is consistent with the typical depletion of $\mathrm{Ti}, \mathrm{Zr}, \mathrm{Y}$, and the REE in alteration halos, and the fact that there are no obvious regions of enrichment in the 504B crustal section. However, the REE pattern of a typical hydrothermal vent fluid has a pronounced light-rare-earth enrichment, and a large (10-20), positive Eu/Eu* anomaly (Michard et al., 1983; Campbell et al., 1988). This pattern could not be produced from the one example given here.

Similar suggestions for $\mathrm{Ti}$ and $\mathrm{Zr}$ in hydrothermal fluids are difficult to make at this time because it is common practice to use titanium collection bottles or syringes when sampling high-temperature hydrothermal vents (e.g., Von Damm et al., 1985; Michard and Al- 


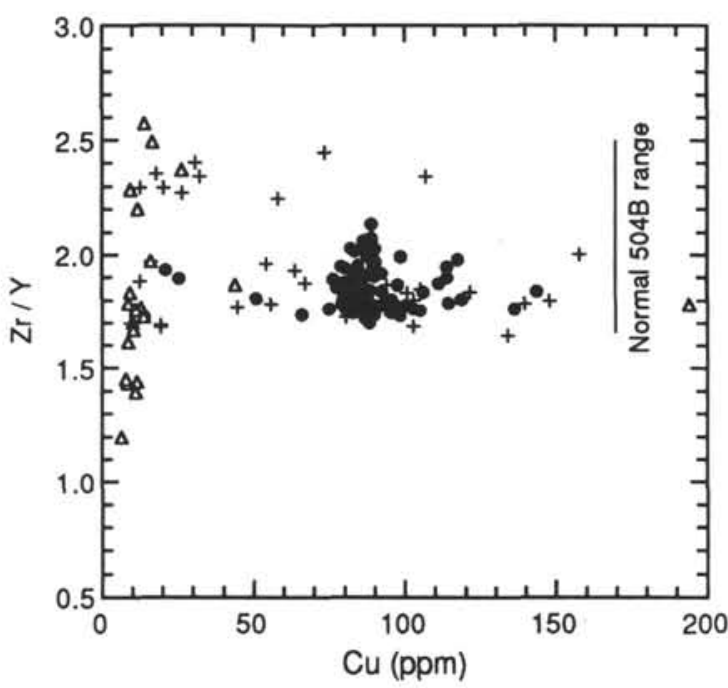

Figure 11. Cu vs. $\mathrm{Zr} / \mathrm{Y}$. The $\mathrm{Zr} / \mathrm{Y}$ ratio decreases slightly at high degrees of alteration, but it is less affected than either $\mathrm{Zr}$ or $\mathrm{Y}$, individually. There is no strong decoupling of these two elements with increased alteration. The samples with $50 \%-95 \%$ alteration and $\mathrm{Zr} / \mathrm{Y}$ values between 2.2 and 2.6 are within the range described by relatively fresh samples from higher levels in the hole. There is no significant evidence of $\mathrm{Ti}, \mathrm{Zr}$, or $\mathrm{Y}$ enrichment resulting from alteration.

barède, 1986). The level of $\mathrm{Ti}$ and $\mathrm{Zr}$ contamination precludes accurate trace element analysis of these elements.

\section{Zinc Depletion With Depth}

The leaching of $\mathrm{Zn}$ and $\mathrm{Cu}$, coupled with the presence of massive sulfide deposits, is a common feature in ophiolite complexes (e.g., Troodos [Richardson et al., 1987] and Semail [Koski et al., 1987]). At the Troodos ophiolite, $\mathrm{Zn}$ and $\mathrm{Cu}$ depletion is strongly linked to the presence of epidosite zones (quartz + epidote rocks) in the lower sections of the sheeted dike complex. The epidosites represent fossil conduits for hydrothermal vents and their associated massive sulfide deposits. Within these zones, $\mathrm{Zn}$ and $\mathrm{Cu}$ are thought to be liberated from the epidosite and surrounding diabase dikes by reactions with hydrothermal fluids at temperatures of $350^{\circ}-400^{\circ} \mathrm{C}$ (Richardson et al., 1987; Schiffman and Smith, 1988). Much of this hydrothermal activity probably occurred as the crustal section moved off-axis and was reheated by the emplacement of hypabyssal stocks (Schiffman and Smith, 1988).

In contrast, at Hole 504B a stockwork-like sulfide-mineralization zone (910-928 mbsf) occurs in the transition from sheeted dikes to the overlying pillow lavas, but large-scale epidosite veins have yet to be identified. Sulfide precipitation is thought to have resulted from hydrothermal fluids (up to $380^{\circ} \mathrm{C}$ ) upwelling through low porosity sheeted dikes and reacting with cooler, down-flowing seawater from the highly porous volcanic section (Alt, 1984; Alt et al., 1985, 1986a; Honnorez et al., 1985). A likely source of hydrothermal $\mathrm{Zn}$ (and $\mathrm{Cu}$ ) for sulfide mineralization zones and ridge-crest black smokers is the slightly to highly altered diabase of the (lower?) sheeted dikes. Typical primary $\mathrm{Zn}$ concentrations at Hole 504B are approximately $70 \pm$ $10 \mathrm{ppm}$ (Fig. 8A), but between 1700 and 2000 mbsf, $\mathrm{Zn}$ is progressively depleted in both "fresh" and highly altered samples. A comparable pattern of $\mathrm{Zn}$ depletion with depth has been observed in the lower section of sheeted diabase dikes of the Josephine ophiolite (Harper et al., 1988) where, as at Troodos, epidosites are thought to be remnants of a circulation system that allowed transport of hydrothermal fluids to ridge-crest black smokers and to sulfide mineralization zones. The lack of extensive epidosite veins at Hole 504B suggests that the hydrothermal circulation system in this section of oceanic crust is less well developed than either the Troodos or Josephine ophiolite. Upward transport of metal-enriched hydrothermal fluids must be accomplished through open, partially mineralized fractures that have yet to develop into large-scale epidosite zones.

High-temperature leaching experiments (Seyfried and Janecky, 1985) help place constraints on the reaction temperatures that are necessary for $\mathrm{Zn}$ and $\mathrm{Cu}$ depletion. These experiments indicate that the concentration of $\mathrm{Zn}$ and $\mathrm{Cu}$ in leachate solutions reaches a maximum between $375^{\circ}$ and $400^{\circ} \mathrm{C}$ and then decreases considerably at

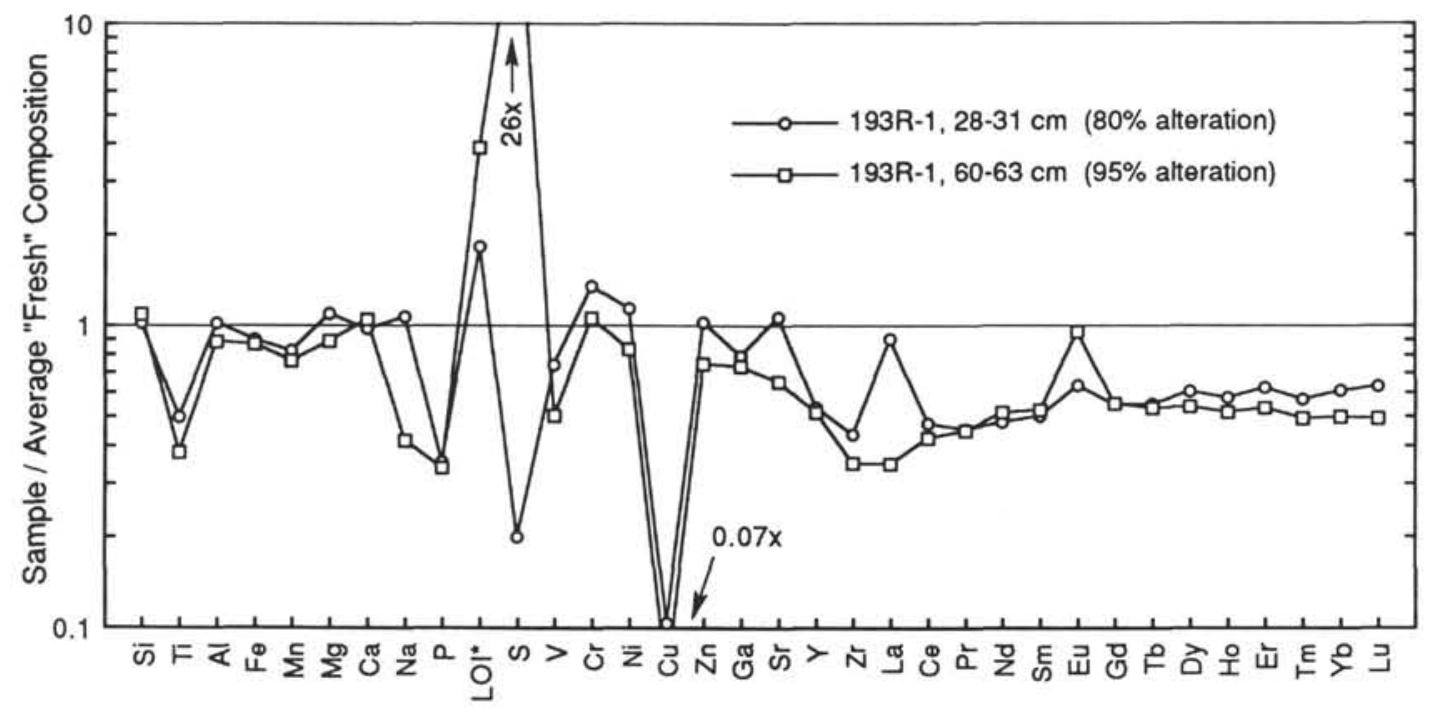

Figure 12. Comparison of "fresh diabase" vs. alteration patches, Unit 220. XRF and ICP-MS analyses of alteration patches are normalized against the average of three samples (140-504B-193R-1, 17-20 cm; -193R-1, 44-46 cm; and -194R-1, 5-9 cm) representing slight to moderate alteration. Four of the five Unit 220 samples were taken within a $45-\mathrm{cm}$ interval in one core section (193R-1); the remaining sample (140-504B-194R-1, 5-9 cm) was located within $10 \mathrm{~cm}$ of the top of the next core. 


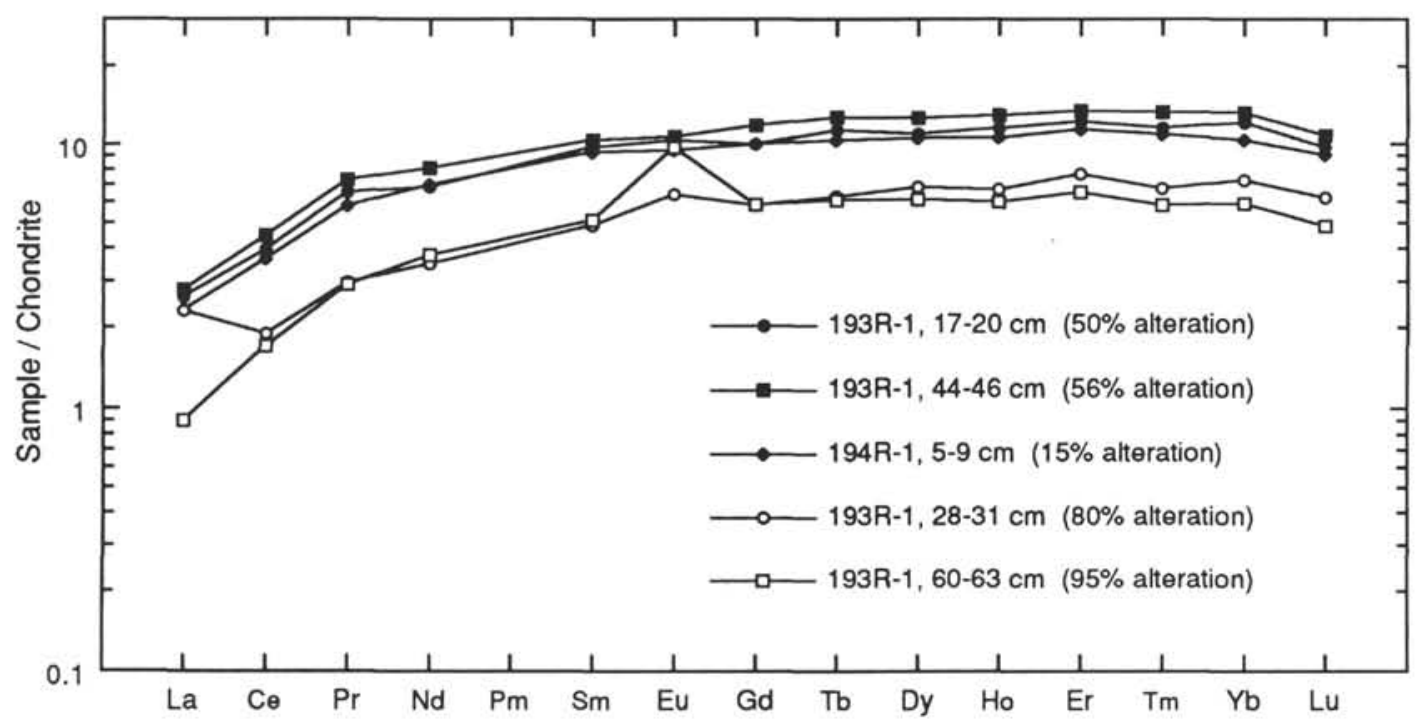

Figure 13. Chondrite-normalized rare-earth element patterns for Unit 220. Chondrite values from Haskin et al. (1968) and Boynton (1989).

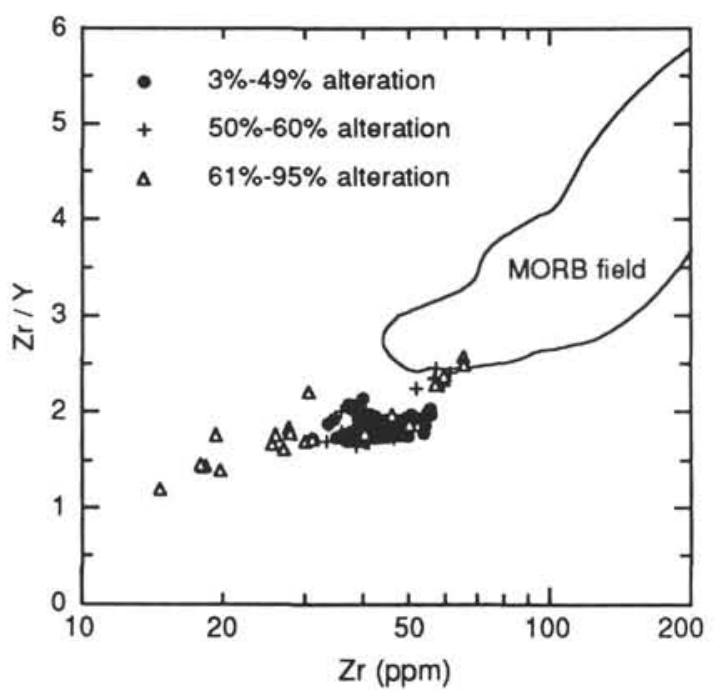

Figure 14. $\mathrm{Zr}$ vs. $\mathrm{Zr} / \mathrm{Y}$. Highly altered samples add a considerable amount of scatter to a relatively well defined Leg $137 / 140$ field. The higher $\mathrm{Zr} / \mathrm{Y}$ samples are still within normal Hole 504B range, and it is likely that they represent primary igneous signatures. MORB field constructed as in Figure 1.

$425^{\circ} \mathrm{C}$. This temperature range is in broad agreement with estimates from the dike section of Hole 504B. Alt et al. (1989b) estimated a temperature range of $250^{\circ}-350^{\circ} \mathrm{C}$ in the Leg 111 section of the dikes based on secondary mineralogy and oxygen isotope data. At greater depths, general increases in the proportions of actinolite replacing clinopyroxene, and the increasing stability of calcic plagioclase over albite reflect rising temperatures with depth that approach uppergreenschist facies conditions in the Leg 140 section (Shipboard Scientific Party, 1992; Laverne et al., this volume).

The behavior of $\mathrm{Cu}$ (Fig. 8C) in the altered diabase is more complex than $\mathrm{Zn}$, and may be related to $\mathrm{Cu}$ being a major component in only one phase (chalcopyrite), whereas $\mathrm{Zn}$ is present in minor amounts in clinopyroxene, chlorite, actinolite, Ti-magnetite as well as sulfides. According to Seyfried and Janecky (1985), Mn and Fe are strongly leached at $425^{\circ} \mathrm{C}$, depending on the $\mathrm{pH}$ of the hydrothermal solution. With this in mind, similar $\mathrm{Mn}$ and Fe depletion zones may become apparent with further penetration of Hole 504B.

\section{CONCLUSIONS}

1. Disregarding highly mobile elements that are affected by subsolidus alteration, the compositions of basalt and diabase samples recovered on Leg 140 are virtually identical to those of the volcanic section, transition zone, and the upper sheeted dike complex previously sampled by Legs $69,70,83$, and 111. All samples analyzed in this study are classified as Group D compositions (e.g., Autio and Rhodes, 1983; Kempton et al., 1985), with compatible element signatures that are similar to moderately evolved MORB, but with unusual depletion in incompatible elements, and elevated levels of $\mathrm{CaO} / \mathrm{Na}_{2} \mathrm{O}$ ratios. These features are consistent with the multistage melting of a normal MORB mantle source (e.g., Duncan and Green, 1980), followed by extensive crystal fractionation (Autio and Rhodes, 1983; Autio, 1984; Kempton et al., 1985).

2. Considering all of Hole 504B, no significant trends indicating incompatible element enrichment or depletion of source magmas over time are apparent. Patterns of phenocryst addition and/or influx of slightly more primitive magma are identifiable at various levels in the sheeted dikes, but broad trends signifying increased or decreased differentiation are absent.

3. A broad zone of $\mathrm{Zn}$ depletion from about 1700 to $2000 \mathrm{mbsf}$ resulting from high-temperature $\left(375^{\circ}-400^{\circ} \mathrm{C}\right)$ leaching is a likely source of hydrothermal $\mathrm{Zn}$ for sulfide mineralization zones near the sheeted dike/transition zone interface, and ridge-crest black smokers. Manganese and iron may begin to exhibit similar depletion trends at greater depths as temperatures increase $\left(\geq 425^{\circ} \mathrm{C}\right)$. In contrast, Cu can be virtually absent locally (down to $7 \mathrm{ppm}$ ), but there is no systematic variation with depth.

4. Areas of intense alteration exhibit significant depletions in $\mathrm{Ti}$, $\mathrm{Zr}$, Y, and REE. These localized (centimeter to meter scale) alteration patches can create significant compositional scatter in igneous trends featuring these elements, if not accounted for. The patches are generally associated with regions near fluid flow, such as veins or void space, and could be a significant source of $Y$ and REE to the typically enriched hydrothermal fluids at ridge-crest vents. 


\section{ACKNOWLEDGMENTS}

I thank L.K Autio and J.M. Rhodes for numerous discussions and debates concerning the petrogenesis of CRRZ basalts. Their input has been invaluable. I also thank J.A. Pearce and J.L. Karsten for prompt reviews that added clarity and depth to the manuscript. This study was supported by a grant from JOI/USSAC to J.W. Sparks and G.A. Mahood.

\section{REFERENCES}

Alt, J.C., 1984. The structure, chemistry, and evolution of a submarine hydrothermal system, DSDP Site 504 [Ph.D. dissert.]. Univ. of Miami, Miami, FL.

Alt, J.C., Anderson, T.F., and Bonnell, L., 1989a. Geochemistry of sulfur in a $1.3 \mathrm{~km}$ section of hydrothermally altered oceanic crust, DSDP Hole 504B. Geochim. Cosmochim. Acta, 53:1011-1023.

Alt, J.C., Anderson, T.F., Bonnell, L., and Muehlenbachs, K., 1989b. Mineralogy, chemistry, and stable isotopic compositions of hydrothermally altered sheeted dikes: ODP Hole 504B, Leg 111. In Becker, K., Sakai, H., et al., Proc. ODP, Sci. Results, 111: College Station, TX (Ocean Drilling Program), 27-40.

Alt, J.C., and Chaussidon, M., 1989. Ion microprobe analyses of the sulfur isotopic composition of sulfides in hydrothermally altered rocks, DSDP/ ODP Hole 504B. In Becker, K., Sakai, H., et al., Proc. ODP, Sci. Results, 111: College Station, TX (Ocean Drilling Program), 41-45.

Alt, J.C., Dick, H.J.B., Erzinger, J., Stokking, L.B., Agrinier, P., Allerton, S., Boldreel, L.O., Fisk, M.R., Harvey, P.K., Iturrino, G.J., Johnson, K.T.M., Kepezhinskas, P., Kelley, D.S., Laverne, C., Marton, F.C., McNeill, A.W., Naslund, H.R., Pariso, J.E., Pertsev, N., Pezard, P., Schandl, E.S., Sparks, J.W., Tartarotti, P., Umino, S., Vanko, D.A., and Zuleger, E., 1992. Hydrothermal alteration of a $2 \mathrm{~km}$ "reference section" of the upper ocean crust, DSDP/ODP Hole 504B. In The Transition From Basalt To Metabasalt. IGCP Project 294, Davis, CA. (Abstract)

Alt, J.C., and Emmermann, R., 1985. Geochemistry of hydrothermally altered basalts: Deep Sea Drilling Project Hole 504B. In Anderson, R.N., Honnorez, J., Becker, K., et al., Init. Repts. DSDP, 83: Washington (U.S. Govt. Printing Office), 249-262.

Alt, J.C., Honnorez, J., Laverne, C., and Emmermann, R., 1986a. Hydrothermal alteration of a $1 \mathrm{~km}$ section through the upper oceanic crust, Deep Sea Drilling Project Hole 504B: mineralogy, chemistry, and evolution of seawater-basalt interactions. J. Geophys. Res., 91:10309-10335.

Alt, J.C., Laverne, C., and Muehlenbachs, K., 1985. Alteration of the upper oceanic crust: mineralogy and processes in Deep Sea Drilling Project Hole 504B, Leg 83. In Anderson, R.N., Honnorez, J., Becker, K., et al., Init. Repts. DSDP, 83: Washington (U.S. Govt. Printing Office), 217-247.

Alt, J.C., Muehlenbachs, K., and Honnorez, J., 1986b. An oxygen isotopic profile through the upper kilometer of the oceanic crust, DSDP Hole 504B. Earth Planet. Sci. Lett., 80:217-229.

Autio, L.K., 1984. Compositional diversity of mid-ocean ridge basalts: an experimental and geochemical study with emphasis on the depleted Costa Rica Rift Zone basalts [Ph.D. dissert.]. Univ. of Massachusetts, Amherst, MA.

Autio, L.K., and Rhodes, J.M., 1983. Costa Rica Rift Zone basalts: geochemical and experimental data from a possible example of multistage melting. In Cann, J.R., Langseth, M.G., Honnorez, J., Von Herzen, R.P., White, S.M., et al., Init. Repts. DSDP, 69: Washington (U.S. Govt. Printing Office), 729-745.

Autio, L.K., Sparks, J.W., and Rhodes, J.M., 1989. Geochemistry of Leg 111 basalts: intrusive feeders for highly depleted pillows and flows. In Becker, K., Sakai, H., et al., Proc. ODP, Sci. Results, 111: College Station, TX (Ocean Drilling Program), 3-16.

Barrett, T.J., 1983. Strontium- and lead-isotope composition of some basalts from Deep Sea Drilling Project Hole 504B, Costa Rica Rift, Legs 69 and 70. In Cann, J.R., Langseth, M.G., Honnorez, J., Von Herzen, R.P.,

*Abbreviations for names of organizations and publications in ODP reference lists follow the style given in Chemical Abstracts Service Source Index (published by American Chemical Society).
White, S.M., et al., Init. Repts. DSDP, 69: Washington (U.S. Govt. Printing Office), 643-650.

Barrett, T.J., and Friedrichsen, H., 1982. Strontium and oxygen isotopic composition of some basalts from Deep Sea Drilling Project Hole 504B, Costa Rica Rift, Legs 69, 70. Earth Planet. Sci. Lett., 60:27-38.

1983. Oxygen- and hydrogen-isotope composition of some basalts from Deep Sea Drilling Project Hole 504B, Costa Rica Rift, Legs 69 and 70. In Cann, J.R., Langseth, M.G., Honnorez, J., Von Herzen, R.P., White, S.M., et al., Init. Repts. DSDP, 69: Washington (U.S. Govt. Printing Office), 637-642.

Basaltic Volcanism Study Project, 1981. Basaltic Volcanism on the Terrestrial Planets: New York (Pergamon Press).

Belyi, V.M., Migdisov, A.A., Barskaya, N.V., and Grinenko, V.A., 1983. Sulfur-isotope composition of hydrothermal sulfides from Hole 504B, Deep Sea Drilling Project Leg 70, Costa Rica Rift. In Cann, J.R., Langseth, M.G., Honnorez, J., Von Herzen, R.P., White, S.M., et al., Init. Repts. DSDP, 69: Washington (U.S. Govt. Printing Office), 619-627.

Bienvenu, P., Bougault, H., Joron, J.L., Treuil, M., and Dmitriev, L., 1990. MORB alteration: rare-earth element/non-rare-earth hygromagmaphile element fractionation. Chem. Geol., 82:1-14.

Boynton, W.V., 1989. Cosmochemistry of the rare earth elements: condensation and evaporation. In Lipin, B.R., and McKay, G.A. (Eds.), Geochemistry and Mineralogy of the Rare Earth Elements. Rev. Mineral., 21:124.

Bryan, W.B., Thompson, G., Frey, F.A., and Dickey, J.S., 1976. Inferred settings and differentiation in basalts from the Deep Sea Drilling Project. $J$. Geophys. Res., 81:4285-4304.

Campbell, A.C., Palmer, M.R., Klinkhammer, G.P., Bowers, T.S., Edmond, J.M., Lawrence, J.R., Casey, J.F., Thompson, G., Humphris, S., Rona, P., and Karson, J.A., 1988. Chemistry of hot springs on the Mid-Atlantic Ridge. Nature, 335:514-519.

Cann, J.R., 1970. Rb, Sr, V, Zr, and $\mathrm{Nb}$ in some ocean floor basaltic rocks. Earth Planet. Sci. Lett., 10:7-11.

Coleman, R.G., 1977. Ophiolites: Ancient Oceanic Lithosphere: New York (Springer-Verlag).

Dick, H.J.B., Erzinger, J.A., Stokking, L.B., Agrinier, P., Allerton, S., Alt, J.C., Boldreel, L.O., Fisk, M.R., Harvey, P.K.H., Iturrino, G.J., Kelley, D.S., Kepezhinskas, P.K., Laverne, C., Marton, F., McNeill, A.W., Naslund, H.R., Pariso, J., Pertsev, N.N., Pezard, P., Schandl, E.S., Sparks, J.W., Tartarotti, P., Umino, S., Vanko, D.A., and Zuleger, E., 1992. ODP drills deepest hole in ocean crust. Eos, 73:537.

Duncan, R.A., and Green, D.H., 1980. Role of multistage melting in the formation of oceanic crust. Geology, 8:22-26.

Emmermann, R., 1985. Basement geochemistry, Hole 504B. In Anderson, R.N., Honnorez, J., Becker, K., et al., Init. Repts. DSDP, 83: Washington (U.S. Govt. Printing Office), 183-199.

Etoubleau, J., Corre, O., Joron, J.L., Bougault, H., and Treuil, M., 1983. Costa Rica Rift: variably depleted basalts in the same hole. In Cann, J.R., Langseth, M.G., Honnorez, J., Von Herzen, R.P., White, S.M., et al., Init. Repts. DSDP, 69: Washington (U.S. Govt. Printing Office), 765-773.

Fisk, M.R., Bence, A.E., and Schilling, J.-G., 1982. Major element chemistry of Galapagos Rift Zone magmas and their phenocrysts. Earth Planet. Sci. Lett., 61:171-189.

Friedrichsen, H., 1985. Strontium, oxygen, and hydrogen isotope studies on primary and secondary minerals in basalts from the Costa Rica Rift, Deep Sea Drilling Project Hole 504B, Leg 83. In Anderson, R.N., Honnorez, J., Becker, K., et al., Init. Repts. DSDP, 83: Washington (U.S. Govt. Printing Office), 289-295.

Green, D.H., Hibberson, W.O., and Jaques, A.L., 1979. Petrogenesis of mid-ocean ridge basalts. In McElhinny, M.W. (Ed.), The Earth: Its Origin, Structure and Evolution: London (Academic Press), 265-290.

Harper, G.D., Bowman, J.R., and Kuhns, R., 1988. A field, chemical and stable isotopic study of subseafloor metamorphism of the Josephine Ophiolite, California-Oregon. J. Geophys. Res., 93:4625-4656.

Haskin, L.A., Frey, F.A., Schmitt, R.A., and Smith, R.H., 1968. Meteoric, solar, and terrestrial rare-earth distributions. In Physics and Chemistry of the Earth 7: New York (Pergamon), 167-321.

Hey, R., Johnson, L., and Lowrie, A., 1977. Recent plate motion in the Galapagos Area. Geol. Soc. Am. Bull., 88:1385-1403.

Hickson, C.J., and Juras, S.J., 1986. Sample contamination by grinding. Can. Mineral., 24:585-589.

Honnorez, J., Alt, J.C., Honnorez, B.M., Laverne, C., Muehlenbachs, K., Saltzman, E., and Ruiz, J., 1985. Stockwork-like sulfide mineralization in young oceanic crust: Deep Sea Drilling Project Hole 504B. In Ander- 
son, R.N., Honnorez, J., Becker, K., et al., Init. Repts. DSDP, 83: Washington (U.S. Govt. Printing Office), 263-282.

Honnorez, J., Laverne, C., Hubberten, H.-W., Emmermann, R., and Muehlenbachs, K., 1983. Alteration processes in Layer 2 basalts from Deep Sea Drilling Project Hole 504B, Costa Rica Rift. In Cann, J.R., Langseth, M.G., Honnorez, J., Von Herzen, R.P., White, S.M., et al., Init. Repts. DSDP, 69: Washington (U.S. Govt. Printing Office), 509-546.

Hubberten, H.-W., 1983. Sulfur content and sulfur isotopes of basalts from the Costa Rica Rift (Hole 504B, Deep Sea Drilling Project Legs 69 and 70). In Cann, J.R., Langseth, M.G., Honnorez, J., Von Herzen, R.P., White, S.M., et al., Init. Repts. DSDP, 69: Washington (U.S. Govt. Printing Office), 629-635.

Hubberten, H.-W., Emmermann, R., and Puchelt, H., 1983. Geochemistry of basalts from Costa Rica Rift Sites 504 and 505 (Deep Sea Drilling Project Legs 69 and 70). In Cann, J.R., Langseth, M.G., Honnorez, J., Von Herzen, R.P., White, S.M., et al., Init. Repts. DSDP, 69: Washington (U.S. Govt. Printing Office), 791-803.

Humphris, S.E., Morrison, M.A., and Thompson, R.N., 1978. Influence of rock crystallization history upon subsequent lanthanide mobility during hydrothermal alteration of basalts. Chem. Geol., 23:125-137.

Humphris, S.E., and Thompson, G., 1978. Hydrothermal alteration of oceanic basalts by seawater. Geochim. Cosmochim. Acta, 42:107-125.

Ishikawa, T., and Nakamura, E., 1992. Boron isotope geochemistry of the oceanic crust from DSDP Hole 504B. Geochim. Cosmochim. Acta, 56:1633-1639.

Ishizuka, H., 1989. Mineral paragenesis of altered basalts from Hole 504B, ODP Leg 111. In Becker, K., Sakai, H., et al., Proc. ODP, Sci. Results, 111: College Station, TX (Ocean Drilling Program), 61-76.

Joron, J.L., Briqueu, L., Bougault, H., and Treuil, M., 1980. East Pacific Rise, Galapagos spreading center and Siqueiros Fracture Zone hygromagmaphile elements-a comparison with the North Atlantic. In Rosendahl, B.R., Hekinian, R., et al., Init. Repts. DSDP, 54: Washington (U.S. Govt. Printing Office), 725-735.

Kawahata, H., and Furuta, T., 1985. Sub-sea-floor hydrothermal alteration in the Galapagos spreading center. Chem. Geol., 49:259-274.

Kempton, P.D., 1985. An interpretation of contrasting nucleation and growth histories from the petrographic analysis of pillow and dike chilled margins, Hole 504B, Deep Sea Drilling Project, Leg 83. In Anderson, R.N., Honnorez, J., Becker, K., et al., Init. Repts. DSDP, 83: Washington (U.S. Govt. Printing Office), 165-181.

Kempton, P.D., Autio, L.K., Rhodes, J.M., Holdaway, M.J., Dungan, M.A. and Johnson, P., 1985. Petrology of basalts from Hole 504B, Deep Sea Drilling Project, Leg 83. In Anderson, R.N., Honnorez, J., Becker, K., et al., Init. Repts. DSDP, 83: Washington (U.S. Govt. Printing Office), 129 164.

Koski, R.A., Haymon, R.M., and Abrams, M., 1987. Ridge-crest and offridge hydrothermal-discharge zones mapped beneath massive sulfide deposits in the Samail Ophiolite, Northern Oman. Eos, 68:1545. (Abstract)

Kusakabe, M., Shibata, T., Yamamoto, M., Mayeda, S., Kagami, H., Honma, H., Masuda, H., and Sakai, H., 1989. Petrology and isotope characteristics $(\mathrm{H}, \mathrm{O}, \mathrm{S}, \mathrm{Sr}$, and $\mathrm{Nd})$ of basalts from Ocean Drilling Program Hole 504B, Leg 111, Costa Rica Rift. In Becker, K., Sakai, H., et al., Proc. ODP, Sci. Results, 111: College Station, TX (Ocean Drilling Program), $47-60$.

Lonsdale, P., and Klitgord, K.D., 1978. Structure and tectonic history of the eastern Panama Basin. Geol. Soc. Am. Bull., 89:981-999.

Ludden, J., and Thompson, G., 1979. An evaluation of the behavior of rareearth elements during the weathering of sea-floor basalts. Earth Planet. Sci. Lett., 43:85-92.

Marsh, N.G., Tarney, J., and Hendry, G.L., 1983. Trace element geochemistry of basalts from Hole 504B, Panama Basin, Deep Sea Drilling Project Legs 69 and 70. In Cann, J.R., Langseth, M.G., Honnorez, J., Von Herzen, R.P., White, S.M., et al., Init. Repts. DSDP, 69: Washington (U.S. Govt. Printing Office), 747-763.

Mattey, D.P., and Muir, I.D., 1980. Geochemistry and mineralogy of basalts from the Galapagos Spreading Center, Deep Sea Drilling Project Leg 54. In Rosendahl, B.R., Hekinian, R., et al., Init. Repts. DSDP, 54: Washington (U.S. Govt. Printing Office), 755-771.

Michard, A., and Albarède, F., 1986. The REE content of some hydrothermal fluids. Chem. Geol., 55:51-60.

Michard, A., Albarède, F., Michard, G., Minster, J.F., and Charlou, J.L., 1983. Rare-earth and uranium in high-temperature solutions from East Pacific Rise hydrothermal vent field $\left(13^{\circ} \mathrm{N}\right)$. Nature, 303:795-797.
Mitchell, J.G., and Terrell, D.J., 1985. Downhole variation of potassium, inert gas abundance, and apparent $\mathrm{K}-\mathrm{Ar}$ age in basalts from the Costa Rica Rift, Hole 504B, Leg 83, Deep Sea Drilling Project. In Anderson, R.N., Honnorez, J., Becker, K., et al., Init. Repts. DSDP, 83: Washington (U.S. Govt. Printing Office), 305-312.

Moore, J.G., 1979. Vesicularity and $\mathrm{CO}_{2}$ in mid-ocean ridge basalt. Nature, 282:250-253.

Natland, J.H., Adamson, A.C., Laverne, C., Melson, W.G., and O'Hearn, T., 1983. A compositionally nearly steady-state magma chamber at the Costa Rica Rift: evidence from basalt glass and mineral data, Deep Sea Drilling Project Sites 501, 504, and 505. In Cann, J.R., Langseth, M.G., Honnorez, J., Von Herzen, R.P., White, S.M., et al., Init. Repts. DSDP, 69: Washington (U.S. Govt. Printing Office), 811-858.

Nielsen, R.L., 1985. EQUIL.FOR: a program for the modeling of low-pressure differentiation processes in natural mafic magma bodies. Comp. Geosci, 11:531-546.

_ 1990. Simulation of igneous differentiation processes. In Nicholl, J., and Russell, J.K. (Eds.), Modern Methods of Igneous Petrology: Understanding Magmatic Processes. Mineral. Soc. Am., Rev. Mineral., 24:65-105.

Noack, Y., Emmermann, R., and Hubberten, H.-W., 1983. Alteration in Site 501 (Leg 68) and Site 504 (Leg 69) basalts: preliminary results. In Cann, J.R., Langseth, M.G., Honnorez, J., Von Herzen, R.P., White, S.M., et al., Init. Repts. DSDP, 69: Washington (U.S. Govt. Printing Office), 497-508.

Norrish, K., and Chappell, B., 1977. X-ray fluorescence spectrometry. In Zussman, J. (Ed.), Physical Methods in Determinative Mineralogy: New York (Academic Press), 201-272.

Norrish, K., and Hutton, J.T., 1969. An accurate X-ray spectrographic method for the analysis of a wide range of geological samples. Geochim. Cosmochim. Acta, 33:431-453.

Pearce, J.A., and Norry, M.J., 1979. Petrogenetic implications of Ti, Zr, Y and $\mathrm{Nb}$ variations in volcanic rocks. Contrib. Mineral. Petrol., 69:33-47.

Price, R.C., Gray, C.M., Wilson, R.E., Frey, F.A., and Taylor, S.R., 1991. The effects of weathering on rare earth element, yttrium and barium abundances in Tertiary basalts from southeastern Australia. Chem. Geol., 93:245-265.

Reynolds, R.C., Jr., 1963. Matrix corrections in trace element analysis by Xray fluorescence: estimation of the mass absorption coefficient by Compton scattering. Am. Mineral., 48:1133-1143.

, 1967. Estimation of mass absorption coefficients by Compton scattering: improvements and extensions of the method. Am. Mineral., 52:1493-1502.

Rhodes, J.M., Dungan, M.A., Blanchard, D.P., and Long, P.E., 1979. Magma mixing at mid-ocean ridges: evidence from basalts drilled near $22^{\circ} \mathrm{N}$ on the Mid-Atlantic Ridge. Tectonophysics, 55:35-61.

Richardson, C.J., Cann, J.R., Richards, H.G., and Cowan, J.G., 1987. Metaldepleted root zones of the Troodos ore-forming hydrothermal systems, Cyprus. Earth Planet. Sci. Lett., 84:243-253.

Schiffman, P., and Smith, B.M., 1988. Petrology and oxygen isotope geochemistry of a fossil seawater hydrothermal system within the Solea Graben, northern Troodos ophiolite, Cyprus. J. Geophys. Res., 93:46124624.

Seyfried, W.E., Jr., and Bischoff, J.L., 1979. Low temperature basalt alteration by seawater: an experimental study at $70^{\circ} \mathrm{C}$ and $150^{\circ} \mathrm{C}$. Geochim. Cosmochim. Acta, 43:1937-1947.

1981. Experimental seawater-basalt interaction at $300^{\circ} \mathrm{C}$ and 500 bars: chemical exchange, secondary mineral formation, and implications for the transport of heavy metals. Geochim. Cosmochim. Acta, 45:135147.

Seyfried, W.E., Jr., and Janecky, D.R., 1985. Heavy metal and sulfur transport during subcritical and super critical hydrothermal alteration of basalt: influence of fluid pressure and basalt composition and crystallinity. Geochim. Cosmochim. Acta, 49:2545-2560.

Sharaskin, A.Y., Migdisov, A.A., Rostschina, I.A., and Miklishansky, A.Z., 1983. Major- and trace-element chemistry of Hole 504B basalts and their alteration products (Costa Rica Rift, Deep Sea Drilling Project Leg 70). In Cann, J.R., Langseth, M.G., Honnorez, J., Von Herzen, R.P., White, S.M., et al., Init. Repts. DSDP, 69: Washington (U.S. Govt. Printing Office), 775-789.

Shimizu, H., Mori, K., and Masuda, A., 1989. REE, Ba, and Sr abundances and $\mathrm{Sr}, \mathrm{Nd}$, and $\mathrm{Ce}$ isotopic ratios in Hole 504B basalts, ODP Leg 111, Costa Rica Rift. In Becker, K., Sakai, H., et al., Proc. ODP. Sci. Results, 111: College Station, TX (Ocean Drilling Program), 77-83. 
Shipboard Scientific Party, 1992. Site 504. In Dick, H.J.B., Erzinger, J., Stokking, L.B., et al., Proc. ODP, Init. Repts., 140: College Station, TX (Ocean Drilling Program), 37-200.

Srivastava, R.K., Emmermann, R., and Puchelt, H., 1980. Petrology and geochemistry of basalts from Deep Sea Drilling Project Leg 54. In Rosendahl, B.R., Hekinian, R., et al., Init. Repts. DSDP, 54: Washington (U.S. Govt. Printing Office), 671-693.

Thompson, G., and Bankston, D.C., 1970. Sample contamination from grinding and sieving determined by emission spectroscopy. Appl. Spectros., $24: 210-219$.

Tual, E., Jahn, B.M., Bougault, H., and Joron, J.L., 1985. Geochemistry of basalts from Hole 504B, Leg 83, Costa Rica Rift. In Anderson, R.N., Honnorez, J., Becker, K., et al., Init. Repts. DSDP, 83: Washington (U.S. Govt. Printing Office), 201-214.

Von Damm, K.L., Edmond, J.M., Grant, B., Measures, C.I., Walden, B., and Weiss, R.F., 1985. Chemistry of submarine hydrothermal solutions at $21^{\circ} \mathrm{N}$, East Pacific Rise. Geochim. Cosmochim. Acta, 49:2197-2220.

Von Herzen, R.P., Robinson, P.T., et al., 1991. Proc. ODP, Sci. Results, 118: College Station, TX (Ocean Drilling Program).
Walker, D., 1973. Behavior of X-ray mass absorption coefficients near absorption edges: Reynold's method revisited. Am. Mineral., 58:10691072.

Willis, J.P., 1989. Compton scatter and matrix correction for trace element analysis of geological materials. In Ahmedali, S.T. (Ed.), X-ray Fluores cence Analysis in the Geological Sciences; Advances in Methodology. Geol. Assoc. Can. Short Course, 7:91-140.

Winchester, J.A., and Floyd, P.A., 1976. Geochemical magma type discrimination: application to altered and metamorphosed basic igneous rocks. Earth Planet. Sci. Lett., 28:459-469.

Date of initial receipt: 20 April 1993

Date of acceptance: 13 January 1994

Ms 137/140SR-021 